\title{
Analysis of Spacecraft Disposal Solutions from LPO to the Moon with High Order Polynomial Expansions
}

\author{
Massimo Vetrisano and Massimiliano Vasile \\ Advanced Space Concepts Laboratory, Department of Mechanical and Aerospace \\ Engineering, University of Strathclyde, James Weir Building, 75 Montrose Street, G1 1XQ \\ Glasgow, UK; t:+441415482326f:+441415525105. \\ \{massimo.vetrisano\}\{massimiliano.vasile\}@strath.ac.uk
}

\begin{abstract}
This paper presents the analysis of disposal trajectories from libration point orbits to the Moon under uncertainty. The paper proposes the use of polynomial chaos expansions to quantify the uncertainty in the final conditions given an uncertainty in initial conditions and disposal manoeuvre. The paper will compare the use of polynomial chaos expansions against high order Taylor expansions computed with point-wise integration of the partials of the dynamics, the use of the covariance matrix propagated using a unscented transformation and a standard Monte Carlo simulation. It will be shown that the use of the ellipsoid of uncertainty, that corresponds to the propagation of the covariance matrix with a first order Taylor expansions, is not adequate to correctly capture the dispersion of the trajectories that can intersect the Moon. Furthermore, it will be shown that polynomial chaos expansions better represent the distribution of the final states compared to Taylor expansions of equal order and are comparable to a full scale Monte Carlo simulations but at a fraction of the computational cost.
\end{abstract}

\section{Introduction}

In a number of cases in space flight dynamics, the chaotic nature of the interaction between the spacecraft and the surrounding environment during orbital transfers and the low confidence level in key spacecraft components (such as propulsion and attitude control) imposes expensive and time consuming ground support campaigns to ascertain that the spacecraft will follow the designed trajectory. In the specific case of disposal manoeuvres, this is undesirable, considering that this is the last manoeuvre available. For this reason, the uncertainty in the states of the spacecraft needs to be properly quantified to maximise the probability of a successful disposal and possibly inform the mission planning that mid-course correction manoeuvres are required. For strongly non-linear and possibly chaotic dynamics, like the one described in this paper, small variations in the initial conditions can result in significantly different trajectories. Therefore, the distribution of the initial states generally maps to a significantly different distribution of the final states. For example, a Gaussian distribution with zero mean and diagonal covariance matrix can map into a distribution, maybe multimodal, that cannot be described only with the first two statistical moments. Furthermore, techniques that change parameterisation to orbital elements to then use the propagation of the covariance matrix are not applicable in the case analysed in this paper (Sabol et al. 2010). Hence, uncertainty quantification methods that can represent multimodal distributions generated by nonlinear dynamics are required. In this work, in particular, we investigate the use of Polynomial Chaos Expansions, PCE, for the quantification of uncertainty in the disposal trajectories from Libration Point Orbits, LPO, to the Moon. 
LPOs are often selected for astrophysics and solar terrestrial missions. As a number of current and future missions are planned to be placed on these orbits, it is critical to properly analyse possible disposal strategies of these spacecraft to mitigate the risk of damage to other space assets in the future. As an example we consider the ESA mission Herschel, launched on 14 May 2009 towards a Lissajous orbit around the outer collinear Lagrange point (L2) of the Sun-Earth System. Herschel is a multi-user observatory mission, dedicated to perform astronomical observations in the far-infrared and sub-millimetre wavelength range. Given the limited amount of propellant and the relatively short flight time, the envisaged end-of-life strategy foresees a disposal manoeuvre into a lunar impacting trajectory (Colombo et al. 2015a). At the end of life, the spacecraft can rely on a limited ground support, thus it is assumed that the initial conditions of the disposal trajectory will only be known with a low degree of confidence. Thus the objective of the uncertainty analysis is to predict the probability of an impact with the Moon, post execution of the disposal manoeuvre.

A straightforward way to fully capture the nonlinearities of this problem is to use a direct Monte Carlo Simulation, MCS. Given a quantity of interest, derived from the outputs of a simulation (or system response), and its relationship with some stochastic variables, the inputs to the simulation, Monte Carlo Simulations quantify the uncertainty in the quantity of interest by generating a sufficient number of independent realisation of the outputs for a set of samples of the inputs drawn from a prescribed probability distribution. For each sample the input is fixed and the simulation is deterministic. Then, one collects an ensemble of realisations and extracts statistical information on the quantity of interest. Although MCS is straightforward to apply, as it only requires repetitive executions of deterministic simulations, typically a large number of them is needed to converge to the correct distribution of the quantity of interest.

In the past two decades, extensive efforts have been devoted to the development of numerical methods that can improve the quantification of uncertainty without resorting to expensive Monte Carlo Simulations. If only the first two statistical moments are of interest and one can introduce the further hypothesis that the inputs are normally distributed, a reduced number of samples can be simulated using an Unscented Transformation, UT, (Julier et al. 1995) or a univariate reduced quadrature (Padulo et al. 2011).

When the outputs are differentiable with respect to the inputs, statistical moments can be derived from a Taylor expansion of the outputs. Example of this method, commonly known as Taylor-based method of moments, can be found in (Parkinson et al. 1993) and (Lewis and Parkinson 1994). More recently, in 2006, Park and Scheeres proposed a method that derives a higher order Taylor series expansions of the final states given a variation in the initial states. This method propagates the partials of the dynamics, up to an arbitrary order, to obtain the so called State Transition Tensors (STTs) as an extension of the State Transition Matrix (STM). The STTs map a variation in the initial states into a variation of the states at a later epoch (assuming a forward in time propagation of the trajectory). Once the STTs are available one can derive the distribution of the final states from the distribution of the initial states without the need of further integrations of the dynamic equations. In this paper we will show that one problem with this approach is that if the STTs are computed by integration of the differential equations associated to the partials, the number of propagations grows dramatically with the order of the expansion. On the other hand, high order expansions are required to correctly capture nonlinearities in the system response. It is worth mentioning that high order Taylor expansions and the STTs can be obtained also by redefining the algebraic operators so that they operate directly on Taylor polynomials (Valli et al. 2013). This approach can be classified as intrusive as it requires direct access to the simulation model and a modification 
of the simulation code. In the same class one can find intrusive PCE methods (Ghanem and Spanos 1991) and generalised intrusive polynomial chaos expansions (Xiu and Karniadakis 2002), that do not require the redefinition of the algebra, and the recent development of generalised polynomial algebra (Riccardi et al. 2015). However, in this paper we limit our attention only to methods that do not require a redefinition of the algebra. Arguably the method proposed by Park and Scheeres can be classified as intrusive as it requires direct access to the simulation model. However, when partials are individually propagated, this method is computational equivalent to an approach that builds a representation of the quantity of interest via sampling and simulation.

An alternative to the derivation of the distribution of the outputs from MCS or Taylor expansions is offered by stochastic expansions. In particular, PCEs construct an approximation of the outputs, or their distribution, via a reduced number of properly selected samples. Since 1991, PCEs have been applied to a number of problems in different fields, among which nuclear reactors design (Gilli et al. 2012), structural mechanics (Shi and Ghanem, 2004; Ghanem and Doostan, 2006), fluid dynamics (Knio and Le Maître, 2006; Hosder et al. 2006; Najm 2009), state estimation (Blanchard et al. 2010) etc. For some applications in statistics see also Evans and Swartz (1992). In space flight, the method was applied to uncertainty propagation in the 2-body problem by Cheng et al. (2011) and more recently used by Jones and Alireza (2013) to estimate the collision probability between two satellites. The attractive attributes of this method are that: it does not require access to the simulation model; it allows decoupling simulation fidelity from output representation accuracy, it has strong mathematical foundations and has the ability to produce functional representations of any stochastic quantities (Eldred et al. 2011) at a lower computational cost compared to Monte Carlo Simulations.

In this work we propose the use of PCEs constructed with Hermite polynomial bases using spectral projection and the sparse-grids of Genz and Keister (1996). These sparse-grids fully exploit the orthogonality properties of Hermite polynomials and provide accurate results with a low number of samples when the inputs are assumed to be normally distributed. PCEs will be used to compute the distribution of the final states of the spacecraft at the Moon and derive a statistic on the probability of an impact. Given that this is a new application of PCE to astrodynamics, the paper presents a brief analysis of the accuracy and computation cost of PCEs compared to the use of MCS, STT and UT.

The paper is organised as follows. Section 2 describes the disposal trajectory dynamics for Herschel. Then, Section 2.2 presents the methods that were considered for the uncertainty quantification of the disposal trajectory. In particular, we will review the fundamental concepts underneath MCS and UT, briefly analyse the computational complexity of the generation of the STT and then present our implementation of the PCE. Section 4 compares the different techniques and applies the most suitable one to the study of the impact probability. In particular, in Section 4.1 the paper compares PCE, MCS, UT and STT on the LPO to Moon disposal problem, while in Section 4.2 PCEs are applied to the calculation of the impact rate and dispersion of the final states at the Moon.

\section{Dynamic Model and Reference Solution}

The interest is in predicting the rate of impact on the Moon for a disposal trajectory from a Lagrangian Point Orbit (LPO). Therefore, we first introduce the perturbed equations of motion for a spacecraft moving in the Sun-Earth-Moon system, then introduce the nominal 
disposal trajectory of Herschel, which is currently operating around the Sun - Earth Lagrangian Point L2 and finally define the quantity of interest.

\subsection{Dynamic Model}

The dynamics of the spacecraft is defined in the Earth Centred Ecliptic reference Frame (ECEF) and is subject to the gravity effects of three bodies (Sun, Earth and Moon) the Solar Radiation Pressure (SRP), n-body perturbations and the higher order gravity harmonics of the Moon:

$$
\begin{aligned}
& \dot{\mathbf{r}}=\mathbf{v} \\
& \dot{\mathbf{v}}=-\frac{\mu_{E}}{r^{3}} \mathbf{r}-\mu_{S}\left(\frac{\mathbf{r}_{S s c}}{r_{S s c}^{3}}-\frac{\mathbf{r}_{S E}}{r_{S E}^{3}}\right)-\mu_{M}\left(\frac{\mathbf{r}_{M s c}}{r_{M s c}^{3}}-\frac{\mathbf{r}_{M E}}{r_{M E}^{3}}\right)+\mathbf{a}_{S o l a r}+\mathbf{a}_{p b}+\mathbf{a}_{n m}
\end{aligned}
$$

where $\mathbf{r}_{S s c}$ and $\mathbf{r}_{M s c}$ are distance of Sun and Moon from the spacecraft, respectively, while their positions with respect to the Earth are given $\mathbf{r}_{S E}$ and $\mathbf{r}_{M E}$ respectively; $\mu_{E}, \mu_{S}, \mu_{M}$ are constants of Earth, Sun and Moon; $\mathbf{a}_{\text {solar }}$ is the solar pressure modelled as follows (Kubo-aka and Sengoku, 1999):

$$
\mathbf{a}_{\text {Sun }}=C_{R} \frac{P_{1 A U}}{c_{\text {light }}}\left(\frac{r_{A U}}{r_{S s c}}\right)^{2} \frac{\left(\mathbf{r}_{S s c}\right)}{r_{S s c}} \frac{A}{m_{s c}}=C_{R} S_{S R P}\left(\frac{r_{A U}}{r_{S s c}}\right)^{2} \frac{\left(\mathbf{r}_{S s c}\right)}{r_{S c c}} \frac{A}{m_{s c}}
$$

where $A, m_{s c}$ are the satellite cross section area and mass respectively, $C_{R}$ the radiation pressure coefficient (including the diffusion and reflection) and $S_{s r p}$ is the solar radiation pressure at $1 \mathrm{AU}$ equal to the ratio between the solar constant $P_{1 A U}$ and the speed of light. The acceleration given by all the planets, either than the Earth, is included in the terms $\mathbf{a}_{p b}$ while $\mathbf{a}_{n m}$ is the acceleration given by the higher order terms (with $n, m>0$ since the zero order is already included in the nominal dynamics) of the gravity potential of the Moon. The gravity potential is expanded in standard Legendre polynomials, as defined in Cunningham (1970):

$$
U=\frac{\mu_{M}}{R_{M}} \sum_{n=0}^{\infty} \sum_{m=0}^{n}\left(C_{n m} V_{n m}+S_{n m} W_{n m}\right)
$$

where $R_{M}$ the Moon mean radius, $C_{n m}, S_{n m}$ are the potential coefficients that describe the distribution of the mass within the body. For the Moon the potential coefficients are the ones from 100th-degree gravity model 'LP100K'. This allows an efficient computation of the potentials and resulting perturbation as a function of the Cartesian coordinates in the Moon fixed reference frame.

\subsection{Reference Solution and Quantity of Interest}

The uncertainty analysis starts from a nominal reference disposal trajectory that was designed without accounting for uncertainty. In the ESA study (Colombo et al. 2015b) a number of disposal options were considered. The one used as reference solution in this paper leaves the LPO in November 2011 and has initial conditions $\mathbf{x}=\left[\begin{array}{llllll}887698.5 \mathrm{~km} & 1402038.8 \mathrm{~km} & 387608.7 \mathrm{~km} & -0.0963 \mathrm{~km} / \mathrm{s} & -0.0485 \mathrm{~km} / \mathrm{s} & 0.0744 \mathrm{~km} / \mathrm{s}\end{array}\right]$. 
In the original ESA study the trajectory was propagated for 60 days till impact including solar radiation pressure with $C_{R} \frac{A}{m_{s c}}=0.0075 \frac{\mathrm{m}^{2}}{\mathrm{~kg}}$ and the gravity of the Moon with harmonics terms up to degree $10^{\text {th }}$. Figure 1 shows the nominal disposal trajectory from LPO to the Moon.

This trajectory has a relatively short flight time with no loops around the Moon or the Earth. Therefore, prior to the uncertainty analysis, it is worth assessing the impact of some of the terms in the dynamic model, on the successful completion of the disposal manoeuvre. Neglecting the SRP contribution will cause the spacecraft to impact the surface with about $800 \mathrm{~km}$ error from the nominal impact point. Figure 2a) shows what happens by neglecting the higher order terms of the gravity of the Moon: the difference in position at impact point, in this case, is below $0.1 \mathrm{~km}$. Conversely, by neglecting the planetary n-body perturbations ( $\mathbf{a}_{p b}$ terms of Eq.(1)) the difference can be as high as $17 \mathrm{~km}$ with respect to the nominal trajectory, see Figure 2b).

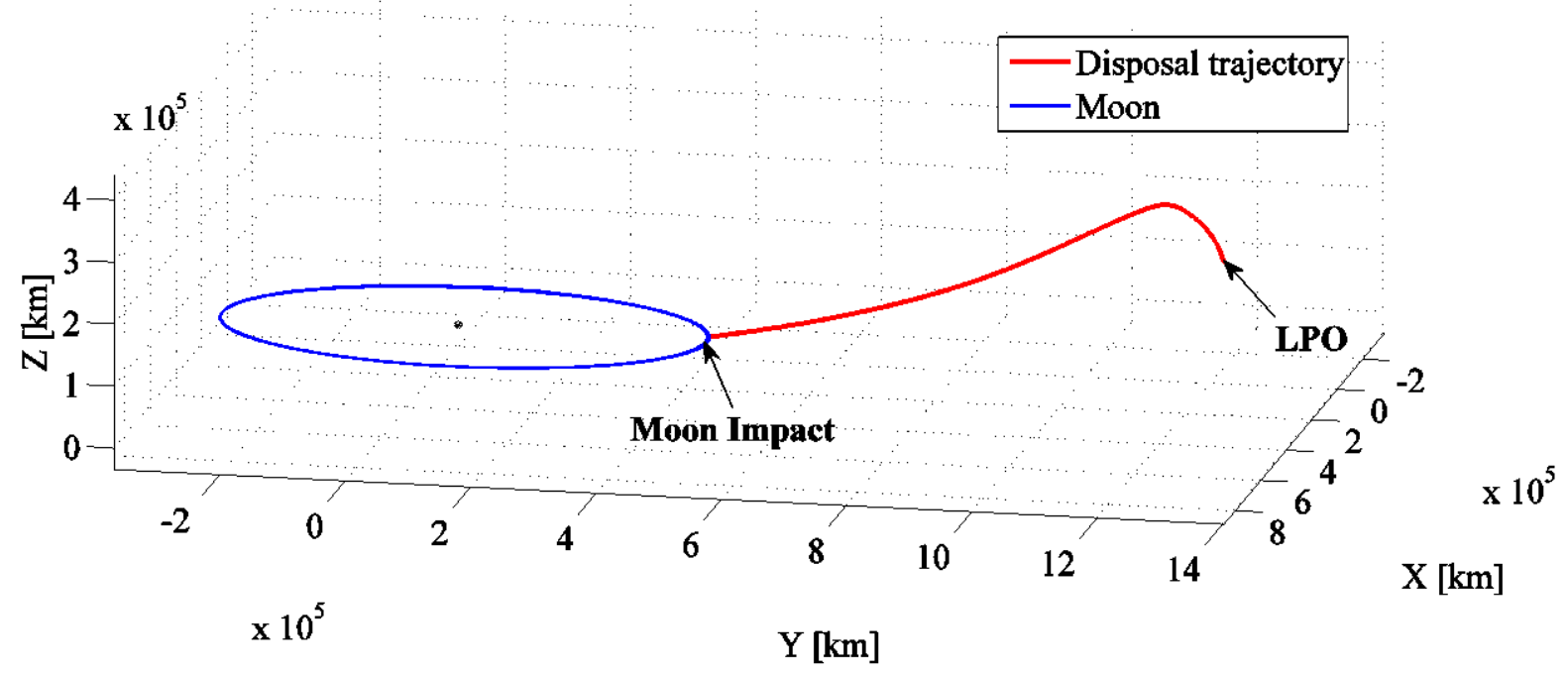

Figure 1. Disposal trajectory towards the Moon for Herschel.

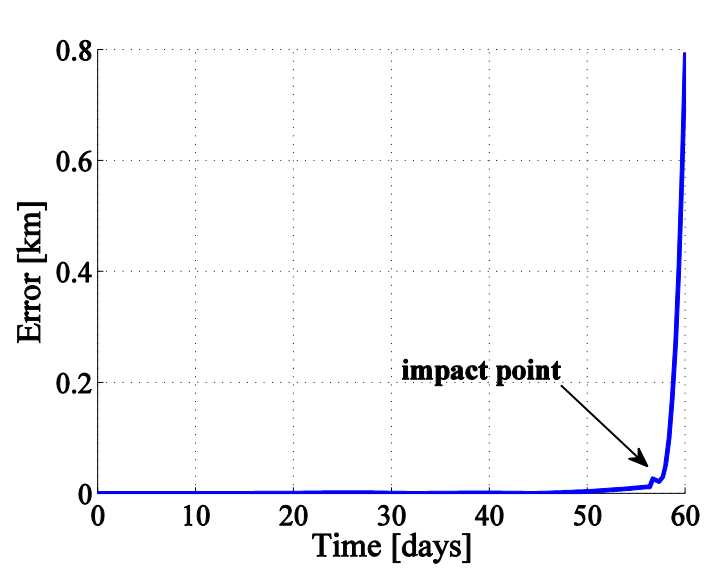

a)

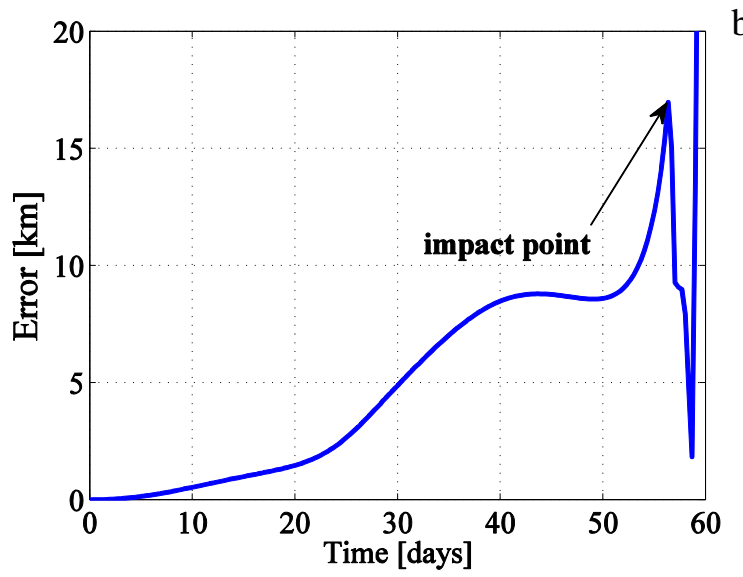

b)

Figure 2. Effects of the non-uniform gravity fields (a) and n-body (b) on the disposal trajectory. 
We can conclude that for the uncertainty analysis the effect of the higher order terms of the gravity of the Moon can be neglected without significant changes in the impact prediction.

\subsubsection{Quantity of Interest}

The quantity of interest is the impact rate, or impact success probability. The impact rate $r_{\text {impact }}$ is calculated as the ratio between the number of virtual trajectories intersecting the Moon surface and the total number of propagated trajectories $N_{\text {samples: }}$ :

$$
r_{\text {impact }}=100 \frac{\sum_{i=1}^{N_{\text {samples }}}\left(r_{\text {perigee }}\left(\boldsymbol{\chi}_{\mathrm{i}}\right) \leq R_{\text {Moon }}\right)}{N_{\text {samples }}} \%
$$

where $r_{\text {perigee }}$ is the radius of the periapsis calculated as a function of the initial uncertain parameters and $R_{\text {Moon }}$ is the Moon mean radius.

\section{Uncertainty Quantification Techniques}

In this section we briefly review three techniques for uncertainties quantification and then introduce our implementation of Polynomial Chaos Expansions. We start first with Monte Carlo Simulations, followed by the Unscented Transformation and the High Order Taylor Expansions, and finally the PCEs.

\subsection{Monte Carlo Simulation}

Monte Carlo Simulations date back as far as Enrico Fermi's study on neutron diffusion, and can be used to derive statistical information via simulation of random samples or to compute multi-dimensional integrals. In uncertainty quantification MCS are used in both ways. The method starts from a probability distribution over the uncertainty space from which samples are drawn. Deterministic simulations are then run for all the samples to derive a quantification of the uncertainty in the output of the simulations.

Under the hypotheses of the Central Limit Theorem, the expected value of a random variable $X$ belongs with probability $\varepsilon$ to the interval

$$
E(X) \in\left[\bar{X}_{n}-\frac{c \bar{\sigma}_{n}}{\sqrt{n}}, \bar{X}_{n}+\frac{c \bar{\sigma}_{n}}{\sqrt{n}}\right]
$$

where $\bar{X}_{n}=\frac{1}{n} \sum_{i}^{n} X_{i}$ and $\bar{\sigma}_{n}^{2}=\frac{1}{n-1} \sum_{i=1}^{n}\left(X_{i}-\bar{X}_{n}\right)^{2}$ and

$$
\varepsilon=\frac{1}{2 \pi} \int_{-c}^{c} e^{-\frac{x^{2}}{2}} d x
$$

From these simple expressions one can deduce that for the mean to converge with confidence 0.95 , the number of samples needs to be: 


$$
\left|E(X)-\bar{X}_{n}\right| \leq 1.96 \frac{\sigma}{\sqrt{n}}
$$

The convergence rate of MCS to the correct mean value is therefore proportional to $1 / \sqrt{n}$. To be noted that the convergence of the mean does not provide any information on the convergence of the distribution or an exact bound on the error.

\subsection{Unscented Transformation}

The Unscented Transformation works on the hypothesis that one can well approximate the posteriori covariance by propagating a limited set of optimally chosen samples (Julier et al. 1995), called sigma points. The set of sigma points $\chi_{k}$, at a certain time step $k$, are defined as:

$$
\boldsymbol{\chi}_{k}=\left\{\begin{array}{cc}
\tilde{\mathbf{x}}_{k} & i=0 \\
\tilde{\mathbf{x}}_{k}+\left(\sqrt{\left(n+\kappa_{u k f}\right) \mathbf{P}_{k}}\right)_{i} & i=1,2, \ldots, n \\
\tilde{\mathbf{x}}_{k}-\left(\sqrt{\left(n+\kappa_{u k f}\right) \mathbf{P}_{k}}\right)_{i} & i=n+1, \ldots, 2 n
\end{array}\right.
$$

where $\chi_{k}$ is a matrix consisting of $(2 n+1)$ vectors $\chi_{k}^{i}$, with $\kappa_{u k f}=\alpha_{u k f}^{2}\left(n+\lambda_{u k f}\right)-n, \kappa_{u k f}$ is a scaling parameter, $\alpha_{u k f}$ determines the extension of these vectors around $\tilde{\mathbf{x}}_{k}$. We set $\alpha_{u k f}$ equal to $10^{-3}$ and $\lambda_{\text {ukf }}$ equal to $3-n$. The sigma points are transformed or propagated through the nonlinear function, the so-called Unscented Transformation, to give:

$$
\boldsymbol{\chi}_{k+1}^{i}=f\left(t, \boldsymbol{\chi}_{k}^{i}\right), \mathrm{i}=0,1, \ldots, 2 \mathrm{n}
$$

The predicted mean of the state vector $\tilde{\mathbf{x}}_{k}$, the covariance matrix $\mathbf{P}_{k}$ can be approximated using the weighted mean and covariance of the transformed vectors:

$$
\begin{gathered}
\boldsymbol{\chi}_{k}^{i}=f\left(t, \boldsymbol{\chi}_{k-1}^{i}\right) \\
\tilde{\mathbf{x}}_{k}=\sum_{i=0}^{2 n} W_{i}^{(m)} \boldsymbol{\chi}_{k}^{i} \\
\mathbf{P}_{k}=\sum_{i=0}^{2 n} W_{i}^{(c)}\left[\boldsymbol{\chi}_{k}^{i}-\tilde{\mathbf{x}}_{k}\right]\left[\boldsymbol{\chi}_{k}^{i}-\tilde{\mathbf{x}}_{k}\right]^{T}
\end{gathered}
$$

where $W_{i}^{(m)}$ and $W_{i}^{(c)}$ are the weighted sample mean and covariance given by:

$$
\begin{gathered}
W_{0}^{(m)}=\kappa /(n+\kappa) \\
W_{0}^{(c)}=\kappa_{u k f} /\left(n+\kappa_{u k f}\right)+\left(1-\alpha_{u k f}^{2}+\beta_{u k f}\right) \\
W_{i}^{(m)}=W_{i}^{(c)}=\kappa_{u k f} /\left[2\left(n+\kappa_{u k f}\right)\right], \quad i=1,2, \ldots, 2 n
\end{gathered}
$$

and $\beta_{u k f}$ is used to incorporate prior knowledge of the distribution with $\beta_{u k f}=2$ (Crassidis and Junkins, 2004). 


\subsection{High-Order Taylor Expansions}

This section briefly reviews the approach proposed by Park and Scheeres (2006) to propagate uncertainty in dynamical systems and highlights some key properties through a simple example. The method expands the variation $\delta \mathbf{x}(t)$ of the states at time $t$ with respect to a reference point $\phi\left(t, \mathbf{x}_{0} ; t_{0}\right)$ in Taylor series of some initial deviation $\delta \mathbf{x}_{0}$. The $s$-th order expansion can be expressed using the Einstein summation convention:

$$
\delta x^{i}(t)=\sum_{p}^{s} \frac{1}{p !} \phi_{\left(t, t_{0}\right)}^{i, \gamma_{1} \cdots \gamma_{p}} \delta x_{0}^{\gamma_{1}} \cdots \delta x_{0}^{\gamma_{p}}
$$

where $\gamma_{1} . . \gamma_{p} \in\{1, \ldots ., n\}$ denotes the $\gamma_{i}$ component of the state vector corresponding to the s-th derivative, $n$ is the number of components of the state vector and:

$$
\phi_{\left(t, t_{0}\right)}^{i, \gamma_{1} \ldots \gamma_{p}}\left(t ; \mathbf{x}_{0} ; t_{0}\right)=\left.\frac{\partial^{p} \phi_{\left(t, t_{0}\right)}^{i}\left(t ; \xi_{0} ; t_{0}\right)}{\partial \xi_{0}^{\gamma_{1}} \cdots \partial \xi_{0}^{\gamma_{p}}}\right|_{\xi_{0}^{\gamma_{j}}=\mathrm{x}_{0}^{\gamma_{j}}}
$$

In this way, a generic trajectory $\mathbf{x}$, whose initial conditions are defined with respect to the reference trajectory as $\mathbf{x}_{0}+\delta \mathbf{x}_{0}$, will evolve as follows:

$$
x^{i}(\mathrm{t})=x_{0}^{i}(\mathrm{t})+\sum_{p}^{s} \frac{1}{p !} \phi_{\left(t, t_{0}\right)}^{i, \gamma_{1} \ldots \gamma_{p}} \delta x_{0}^{\gamma_{1}} \cdots \delta x_{0}^{\gamma_{p}}
$$

The partials of the flow in Eq. (16) form the so called global State Transition Tensors, which map the initial deviations $\delta \mathbf{x}_{0}$ at time $t_{0}$ to the deviation $\delta \mathbf{x}(t)$ at time $t$. For $s=1$, the STTs reduces to the simple state transition matrix. The partials in Eq. (17) can be computed by numerical integration of a set of ordinary differential equations (see Park and Scheeres, 2006a). An example of these differential equations up to the third order follows:

$$
\begin{gathered}
\dot{\phi}^{i, a}=f^{i, \alpha} \phi^{\alpha, a} \\
\dot{\phi}^{i, a b}=f^{i, \alpha} \phi^{\alpha, a b}+f^{i, \alpha \beta} \phi^{\alpha, a} \phi^{\beta, b} \\
\dot{\phi}^{i, a b c}=f^{i, \alpha} \phi^{\alpha, a b c}+f^{i, \alpha \beta}\left(\phi^{\alpha, a} \phi^{\beta, b c}+\phi^{\alpha, a b} \phi^{\beta, c}+\phi^{\alpha, a c} \phi^{\beta, b}\right)+f^{i, \alpha \beta \delta} \phi^{\alpha, a} \phi^{\beta, b} \phi^{\delta, c}
\end{gathered}
$$

where $\alpha, \beta, \in \delta\{1, \ldots, n\}$ and $a, b, c=\{1, \ldots, n\}$ are the indexes for the first, second and third order derivative. $f^{i, \gamma_{1} \ldots \gamma_{p}}$ are the partials of the dynamics and are computed as follows:

$$
f^{i, \gamma_{1} \ldots \gamma_{p}}=\left.\frac{\partial^{p} f^{i}\left(t ; \xi_{0} ; t_{0}\right)}{\partial \xi_{0}^{\gamma_{1}} \cdots \partial \xi_{0}^{\gamma_{p}}}\right|_{\xi_{0}^{\gamma_{j}}=x_{0}^{\gamma_{j}}}
$$

Note that the partial derivatives in Eqs. (17) and (22) are calculated with respect to the nominal trajectory $\phi\left(t, \mathbf{x}_{0} ; t_{0}\right)$ (also equivalent to $\mathbf{x}_{0}(\mathrm{t})$ of Eq. (18)).

If the partials in Eq. (17) are obtained by numerical integration, the calculation of the STTs requires the forward propagation of $\sum_{q=1}^{s+1} 6^{q}$ differential equations starting with initial values $\phi_{\left(t_{0}, t_{0}\right)}^{i, a}=1$, if $i=a$, and zero otherwise. When the order is $s=3$, the 1554 equations need to be integrated simultaneously. Moreover, the computational time and complexity are increased by the numerical evaluations of the analytical partials of the dynamics. In this work, the 
partials in Eq. (22) were computed analytically using the symbolic manipulator in the MATLAB $^{\mathrm{RM}}$ Symbolic Toolbox. As an example, the third order STTs integration, along a 5 day period, considering only Earth, Moon, Sun and light pressure, required approximately 8 hours using a Windows 7 OS $3.16 \mathrm{Ghz}$ Intel ${ }^{(\mathrm{R})} \mathrm{Core}^{(\mathrm{TM})} 2$ Duo CPU. To be noted that the coupled integration of thousands of equations could introduce numerical errors when integrated over a long period of time. For this reason, it is good practice to consider the nominal trajectory and to integrate the STTs over short periods of time, say 1 day to reduce possible numerical errors (Vetrisano et al. 2012). The intermediate STTs are called local STTs. While the global STTs map the deviation at the initial time $t_{0}$ to the deviation at time $t_{k+1}$, the local STTs map the deviation at time $t_{k}$ to the deviation at time $t_{k+1}$.

As an illustrative example, Figure 3 shows the difference between a trajectory with initial conditions $\mathbf{x}_{0}+\delta \mathbf{x}_{0}$ propagated numerically for 5 days (still considering only Earth, Moon, Sun and light pressure) and the corresponding approximation defined in Eq. (18).
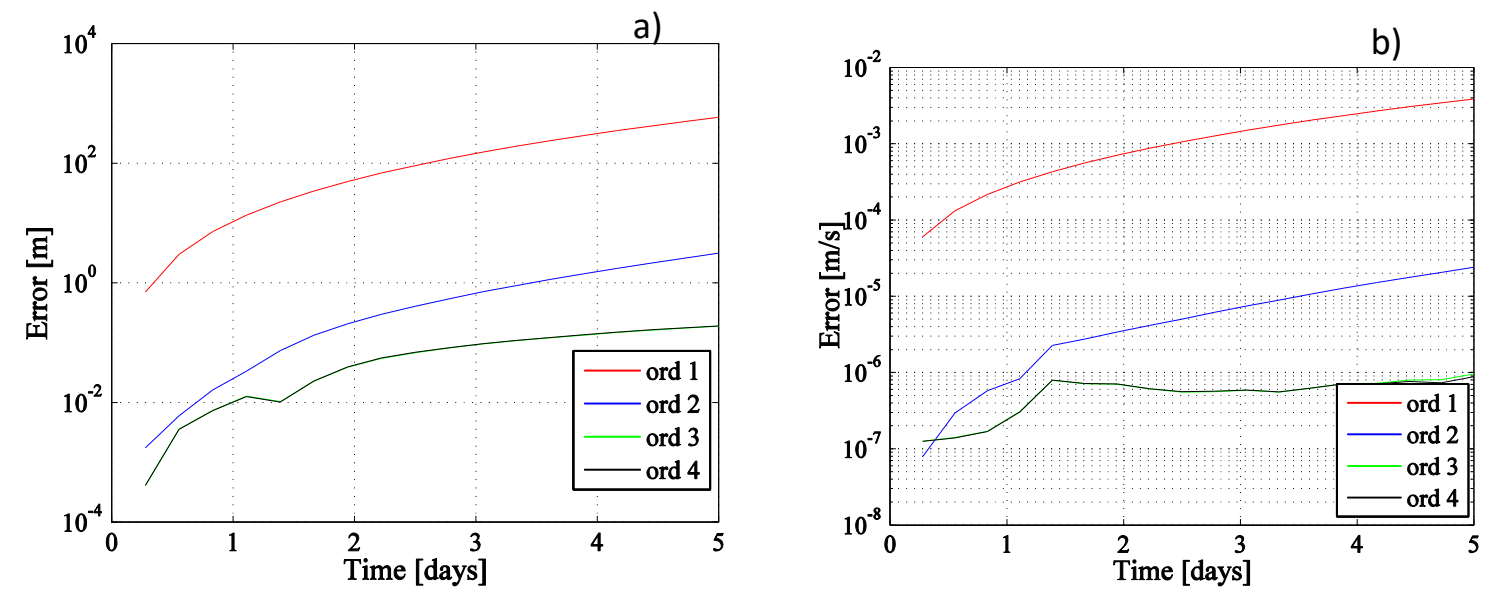

Figure 3. Position (a) and velocity (b) error with local STTs.

For this example the variation of the initial states was set to $\delta \mathbf{x}_{0}=\left[\begin{array}{llllll}2500 \mathrm{~km} & 2500 \mathrm{~km} & 2500 \mathrm{~km} & 0.01 \mathrm{~km} / \mathrm{s} & 0.01 \mathrm{~km} / \mathrm{s} & 0.01 \mathrm{~km} / \mathrm{s}\end{array}\right]^{T}$. The third and fourth order expansions prove to be the most accurate. The difference between approximation (18) and the corresponding trajectory propagated by integrating the equation of motion are reported in Table 1. It is clear that the linear approximation is not enough to correctly capture the evolution of the variated trajectory. The second order expansion offers a better approximation with the third and fourth order expansions showing the lowest error, especially the velocity components. The fact that third and fourth order seem to partially overlap is due to the fact that the trajectory deviated considerably from the reference one, thus the approximation (16) is not able to represent the evolution of the variated state for low orders.

Table 1. Propagation error using STTs.

\begin{tabular}{|l|c|c|c|c|}
\hline Order & 1 & 2 & 3 & 4 \\
\hline $\begin{array}{l}\text { Position } \\
\text { error [m] }\end{array}$ & 585.111 & 3.133 & 0.189 & 0.190 \\
\hline $\begin{array}{l}\text { Velocity } \\
\text { error [m/s] }\end{array}$ & $3.5 \cdot 10^{-3}$ & $2.4 \cdot 10^{-5}$ & $9.6 \cdot 10^{-7}$ & $8.8 \cdot 10^{-7}$ \\
\hline
\end{tabular}


Once the state transition tensors are available for some time interval $\left[t_{k}, t_{k+1}\right]$, the mean and covariance matrix of the relative dynamics at $t_{k}$ can be mapped analytically to $t_{k+1}$ as a function of the probability distribution at $t_{k}$. In the remainder of this paper we will make use of the mean and covariance to compare different methods, therefore, here we briefly summarise the procedure proposed in Park and Scheeres 2006b, and Park, 2007. From $t_{k}$ to $t_{k+1}$ the propagated mean and covariance can be computed as:

$$
\begin{aligned}
& \mathbf{m}_{k+1}^{i}= \phi^{i}\left(t_{k+1} ; \mathbf{m}_{k}\right)+\delta \mathbf{m}_{k+1}^{i}=\phi^{i}\left(t_{k+1} ; \mathbf{m}_{k}\right)+\sum_{p=1}^{s} \frac{1}{p !} \phi_{\left(t_{k+1}, t_{k}\right)}^{i \cdot \gamma_{1} \cdots \gamma_{p}} E\left[\delta x_{k}^{\gamma_{1}} \cdots \delta x_{k}^{\gamma_{p}}\right] \\
& P_{k+1}^{i j}=E\left[\left(\delta x_{k+1}^{i}-\delta m_{k+1}^{i}\right)\left(\delta x_{k+1}^{j}-\delta m_{k+1}^{j}\right)\right]= \\
& \quad \sum_{p=1}^{s} \sum_{q=1}^{s} \frac{1}{p ! q !} \phi_{\left(t_{k+1}, t_{k}\right)}^{i \cdot \gamma_{1} \cdots \gamma_{p}} \phi_{\left(t_{k+1}, t_{k}\right)}^{i \cdot \varsigma_{1} \cdots \varsigma_{q}} E\left[\delta x_{k}^{\gamma_{1}} \cdots \delta x_{k}^{\gamma_{p}} \delta x_{k}^{\varsigma_{1}} \cdots \delta x_{k}^{\varsigma_{q}}\right]-\delta m_{k+1}^{i} \delta m_{k+1}^{j}
\end{aligned}
$$

where $\left\{\gamma_{i}, \varsigma_{j}\right\} \in\{1, \ldots, n\}$ are the indexes for the different order derivative. If one sticks to the hypothesis of an initial Gaussian distribution, the joint characteristic function for a Gaussian random vector can be defined as (Park and Scheeres, 2006)

$$
\vartheta(\mathbf{u})=E\left[e^{j \mathbf{u}^{T} \mathbf{x}}\right]=\exp \left(j \mathbf{u}^{T} \mathbf{m}-\frac{1}{2} \mathbf{u}^{T} \mathbf{P u}\right)
$$

where $j=\sqrt{-1}$ and the expected higher moments can be computed using:

$$
E\left[\mathbf{x}^{\gamma_{1}} \mathbf{x}^{\gamma_{2}} \cdots \mathbf{x}^{\gamma_{p}}\right]=\left.j^{-p} \frac{\partial^{p} \vartheta(\mathbf{u})}{\partial \mathbf{u}^{\gamma_{1}} \partial \mathbf{u}^{\gamma_{2}} \cdots \partial \mathbf{u}^{\gamma_{p}}}\right|_{\mathbf{u}=0}
$$

\subsection{Polynomial Chaos Expansions}

The PCE method employs a set of orthogonal polynomial functions to approximate the functional form between the system response and each of the inputs (Ghanem and Spanos, 1991; Ghanem and Dham, 1998; Xiu and Karniadakis, 2002; Le Matre et al., 2004; Ghanem and Doostan 2006). The chaos expansion for a component $l$ of a generic response $\boldsymbol{G}$ takes the form:

$$
G_{l}=a_{0} B_{0}+\sum_{i_{1}=1}^{\infty} a_{i_{1}} B_{1}\left(\chi_{i_{1}}\right)+\sum_{i_{1}=1}^{\infty} \sum_{i_{2}=1}^{i_{1}} a_{i_{1} i_{2}} B_{2}\left(\chi_{i_{1}}, \chi_{i_{2}}\right)+\sum_{i_{1}=1}^{\infty} \sum_{i_{2}=1}^{i_{1}} \sum_{i_{3}=1}^{i_{2}} a_{i_{1} i_{2} i_{3}} B_{3}\left(\chi_{i_{1}}, \chi_{i_{2}}, \chi_{i_{3}}\right)+\ldots
$$

where $\chi$ are random inputs and $B_{i}$ is a generic multivariate polynomial. This expression can be simplified by replacing the order-based indexing with a term-based indexing:

$$
G_{l}=\sum_{j=0}^{\infty} \alpha_{l j} \Psi_{j}(\chi)
$$

where there is a one-to-one correspondence between $a_{i_{1} i_{2} i_{3}}$ and $\alpha_{l j}$, and between $B_{n}\left(\chi_{i_{1}}, \chi_{i_{2}}, \ldots, \chi_{i_{v}}\right)$ and $\Psi_{j}(\mathbf{x})$. Each of the $\Psi_{j}(\xi)$ is a multivariate polynomials which involve products of the one-dimensional polynomials. In practice, one truncates the infinite expansion at a finite number of random variables and a finite expansion order, $p$ : 


$$
G_{l} \cong \sum_{j=0}^{p} \alpha_{l j} \Psi_{j}(\chi)
$$

Using Hermite polynomials, a multivariate polynomial $B(\xi)$ of order $n$ is defined from:

$$
B_{n}\left(\chi_{i_{1}}, \chi_{i_{2}}, \ldots, \chi_{i_{v}}\right)=e^{\frac{1}{2} \chi^{T} \chi}(-1)^{n} \frac{\partial^{n}}{\chi_{i_{1}}, \ldots, \chi_{i_{v}}} e^{-\frac{1}{2} \chi^{T} \chi}
$$

which can be shown to be a product of one-dimensional Hermite polynomials involving a multi-index $m_{i}^{j}$ :

$$
B_{n}\left(\chi_{i_{1}}, \chi_{i_{2}}, \ldots, \chi_{i_{v}}\right)=\Psi_{j}(\chi)=\prod_{i=1}^{n} \psi_{m_{i}^{j}}\left(\chi_{i}\right)
$$

For a multivariate polynomial the number of coefficients of the expansions for each uncertain variable is given by $\frac{\left(i_{v}+n\right) !}{i_{v} ! n !}$ which shows that the expansions tend to increase quite rapidly with the number of variables and order. The coefficients of the expansion (19) are here calculated via spectral projection (Eldred et al. 2011). This approach projects the response $\mathbf{G}$ against each basis function using inner products and employs the polynomial orthogonality properties to extract each coefficient. Each coefficient in Eq. (29) is calculated as:

$$
\alpha_{l j}=\frac{\left\langle G_{l}, \Psi_{j}\right\rangle}{\left\langle\Psi_{j}^{2}\right\rangle}=\frac{1}{\left\langle\Psi_{j}^{2}\right\rangle} \int_{\Omega} G \Psi_{j} \rho(\chi) d \chi
$$

where the inner product involves a multi-dimensional integral over the support of the weighting function $\rho(\chi)$. Analytical expressions of the mean and covariance matrix are then available as:

$$
\begin{aligned}
& \boldsymbol{\mu}_{G}=E[\mathbf{G}] \cong \sum_{j=0}^{p} \boldsymbol{\alpha}_{j} E\left[\Psi_{j}\right]=\boldsymbol{\alpha}_{0} \\
& \mathbf{P}_{G}=E\left[\left(\mathbf{G}-\boldsymbol{\mu}_{G}\right)\left(\mathbf{G}-\boldsymbol{\mu}_{G}\right)^{T}\right] \cong \sum_{j=1}^{p} \boldsymbol{\alpha}_{j}\left(\boldsymbol{\alpha}_{j}\right)^{T} E\left[\Psi_{j}^{2}\right]
\end{aligned}
$$

$\boldsymbol{\mu}_{G}$ and $\mathbf{P}_{G}$ are the exact moments of the expansion, which converge to moments of the true response function; the vector $\boldsymbol{\alpha}_{j}$ represents the $j$-th column of the matrix $\boldsymbol{\alpha}$ of components $\alpha_{l j}$. The computation of the multi-dimensional integral can be done using a MCS with lowdiscrepancy sequences or a quadrature formula using Gauss points and weights. The latter, however, requires a full tensor product and a number of points that increases exponentially with the number of dimensions. As proposed in Xiu and Hesthaven (2005), a more attractive choice, is based on sparse grids generated using Smolyak's algorithm (Smolyak, 1963). Smolyak's approach provides a general tool for constructing efficient algorithms able to solve multivariate problems with orders of magnitude reduction in the number of support nodes while giving the same level of approximation as the usual tensor product. In this framework, the work of Genz and Keister (1996) introduced fully symmetric interpolatory integration rules for Smolyak sparse grid of Gauss-Hermite nodes. 


\subsubsection{Gauss-Hermite Cubature rules}

The multi-dimensional integral in Eq. (32) can be approximated as the sum of discrete number of terms:

$$
\int_{\Omega} G_{l} \Psi_{j} \rho(\boldsymbol{\chi}) d \chi \cong \sum_{i=1}^{n g r i d} G_{l}\left(\boldsymbol{\chi}_{i}\right) \Psi_{j}\left(\boldsymbol{\chi}_{i}\right) w\left(\boldsymbol{\chi}_{i}\right)
$$

The set of points $\chi_{i}$ and weights $w\left(\chi_{i}\right)$ are defined by the Gauss-Hermite cubature rule in Genz and Keister (1996). These rules are optimal for the solution of multidimensional integrals over infinite regions with a Gaussian weight function. In the work of Genz and Keister (1996), it is shown that a Gaussian integral for a polynomial of order $n$ can be calculated perfectly using a grid of level $l=2 n+1$. Figure 4 shows a normalised sparse grids, with different level of accuracy, for 3 uncertain parameters $\chi_{1}, \chi_{2}$ and $\chi_{3}$ using Hermite polynomials as bases.

Level 2

Level 3

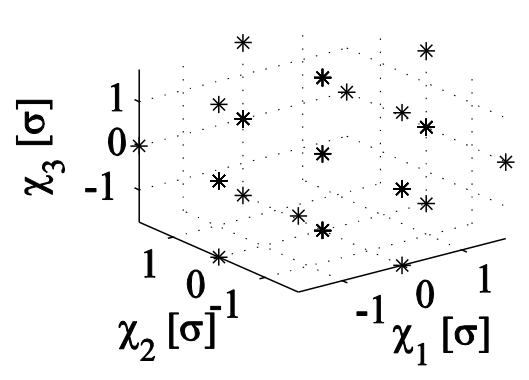

Level 5

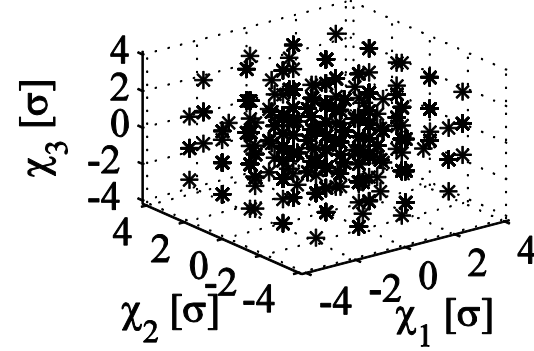

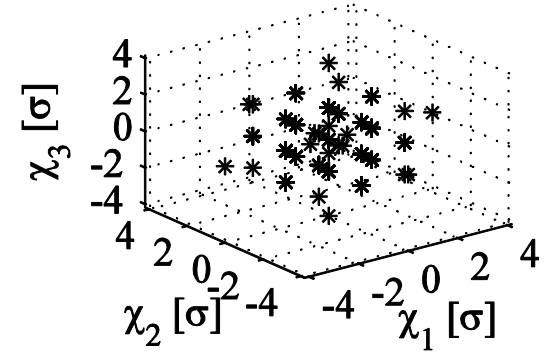

Level 6

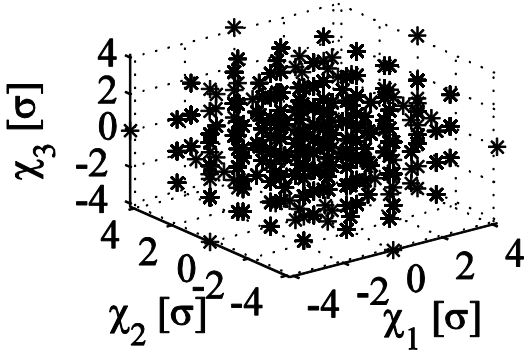

Figure 4. Smoliak grid for Hermits polynomials with different levels of accuracy

As one can see when the level of accuracy is increased the grid is populated by a higher number of samples, which cover also a broader portion of the uncertainty space. For example, the max and min values of the uncertain parameters are respectively $1.7 \sigma$ and $-1.7 \sigma$ for a level 2 grid, while they are $4 \sigma$ and $-4 \sigma$ for a level 6 grid. This gives the possibility to better cover the uncertainty space, generating samples with low associated probability. Table 2 shows the minimum number of points needed to calculate the discrete integrals in Eq. (33), for 6 uncertain variables, as a function of the order of the polynomials. As a reference, the table reports the number of nodes for a classic sparse grid cubature rules (see Heiss and Winschel, 2007), for the same order of expansion. As one can see Genz-Keister grids allows decreasing the number of sampling points as the order of the polynomial increases while maintaining the same accuracy level (see the work of Genz and Keister, 1996 where a detailed comparison was carried out). 
Table 2. Minimum number of points required for calculating Gaussian integrals of a certain order.

\begin{tabular}{|l|c|c|c|c|c|c|}
\hline Order & 1 & 3 & 5 & 7 & 9 & 11 \\
\hline $\begin{array}{l}\text { Genz-Keister } \\
\text { sparse grid }\end{array}$ & 1 & 13 & 73 & 257 & 749 & 2021 \\
\hline $\begin{array}{l}\text { Classic } \\
\text { Sparse grid }\end{array}$ & 1 & 13 & 85 & 389 & 1433 & 4541 \\
\hline
\end{tabular}

\section{Uncertainty Analysis}

This section starts with a comparison among the methods presented in Section 2.2 to select the most suitable alternative to MCS for the case analysed in this paper. Then the study of the probability of impact on the surface of the Moon for the disposal trajectory described in Section 4.2 follows.

\subsection{Comparison}

Given that the uncertainty analysis of disposal trajectories from LPO to the Moon is a new application of PCE in astrodynamics, we first compare the methods presented in Section 2.2 to evaluate their accuracy and computational cost and establish which one provides the best representation of the dispersion of the final states. Section 4.1.1 derives the reference distribution using a Monte Carlo Simulation. Section 4.1.2 compares the ability of each method to correctly predict mean and covariance while Section 4.1.3 tests the ability of STT and PCEs to represent the full distribution versus their computational cost. The dynamical model used in all the tests in this section includes only Earth, Sun and Moon and light pressure and does not consider the full harmonic expansion of the gravity field of the Moon or the n-body perturbation $\mathbf{a}_{p b}$. The dynamic equations were numerically integrated with an explicit, variable step size, Runge-Kutta 4-5 integration method (ode45 function of Matlab ${ }^{\mathrm{TM}}$ ) with respectively a $10^{-9}$ and $10^{-9}$ relative and absolute accuracies.

\subsubsection{Reference Distribution}

In this section we derive the reference distribution used in the following comparisons. The distribution is derived from a full MC simulation using the complete model. The simulation was run for an increasing number samples, from $10^{4}$ to $10^{6}$, to verify the correctness of the predicted distribution.

The uncertain quantities are assumed to be uncorrelated and normally distributed with standard deviation

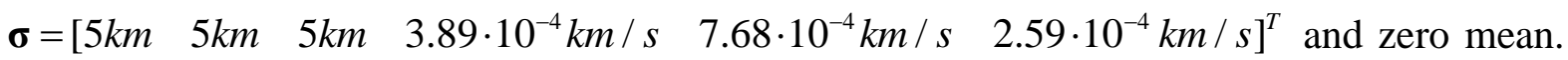
These values are typical position errors for this kind of mission (see Godard et al. 2009) and will be used for the analysis in the remainder of this paper. The variation in velocity represents $1 \%(1 \sigma)$ of the disposal manoeuvre. Note that trajectories are propagated up to 12 hours before the nominal impact because the numerical integration of the STTs beyond that point resulted very difficult due to the proximity to the singularity (represented by the Moon centre) of the equations of motion. 
Figure 5 to Figure 7 show the contour plots of the resulting distributions in the final position projected on the plane $\mathrm{x}-\mathrm{y}$ and the final velocity projected on the plane $\mathrm{x}-\mathrm{z}$. These projections show the most significant nonlinearities and asymmetries in the final distributions. The probability density was computed as the ratio between fraction of particles within a circle centred in each MC sample and the corresponding area. Radii of $100 \mathrm{~km}$ for the position and of $20 \mathrm{~cm} / \mathrm{s}$ for the velocity were used. To be noted that final distributions are not Gaussian, although the initial samples were drawn from a Gaussian distribution.

Figure 5 to Figure 7 show the shape can be described fairly well even with 10,000 samples, although the tails fail to cover all the range which only more samples are able to describe. More samples define low probability density more accurately such that the transition between the different density levels becomes smoother, as in Figure 7.

a)

b)
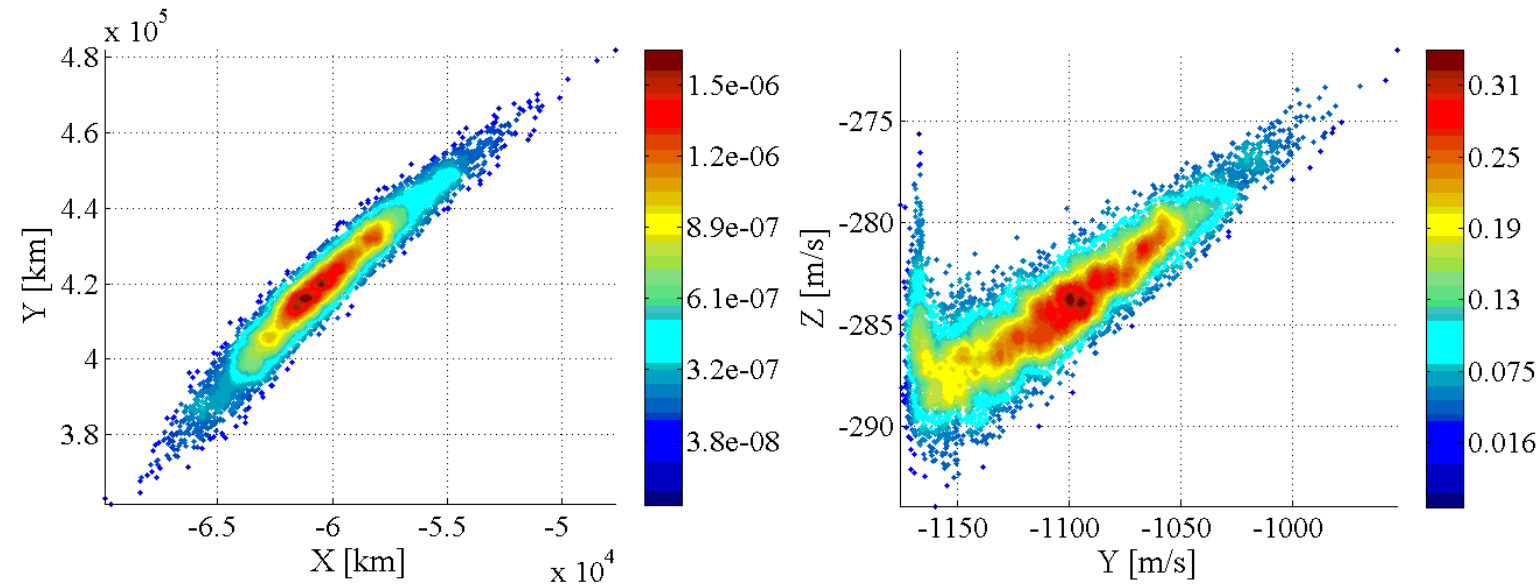

Figure 5. Statistical distribution for Monte Carlo Simulation and 10,000 samples: $x-y$ position distribution (a), $x-z$ velocity distribution (b). The colorbar represents the probability density.

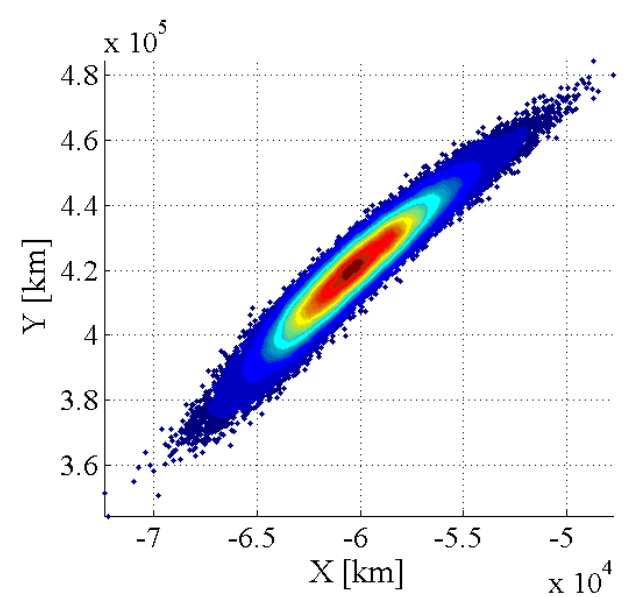

a)
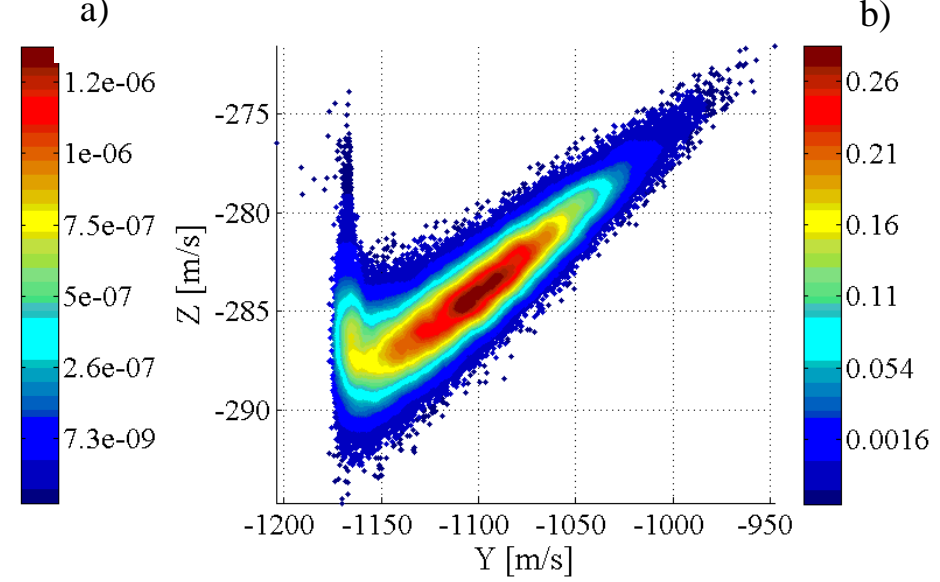

Figure 6. Statistical distribution for Monte Carlo Simulation and 100,000 samples: $x-y$ position distribution (a), $x-z$ velocity distribution (b). The colorbar represents the probability density. 


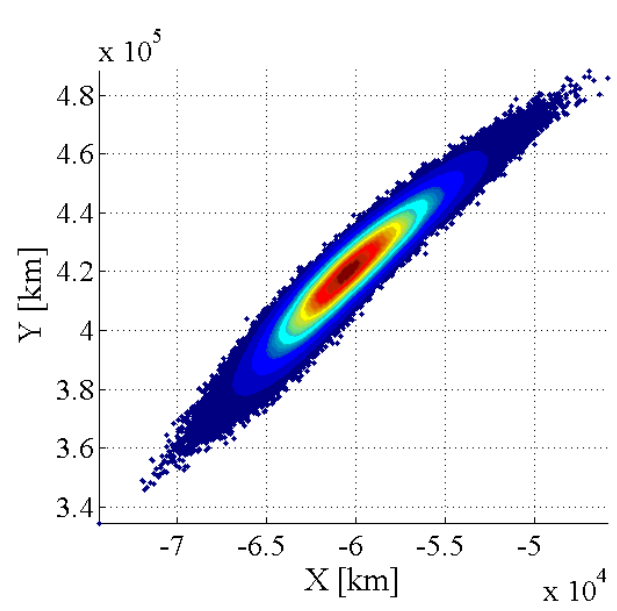

a)
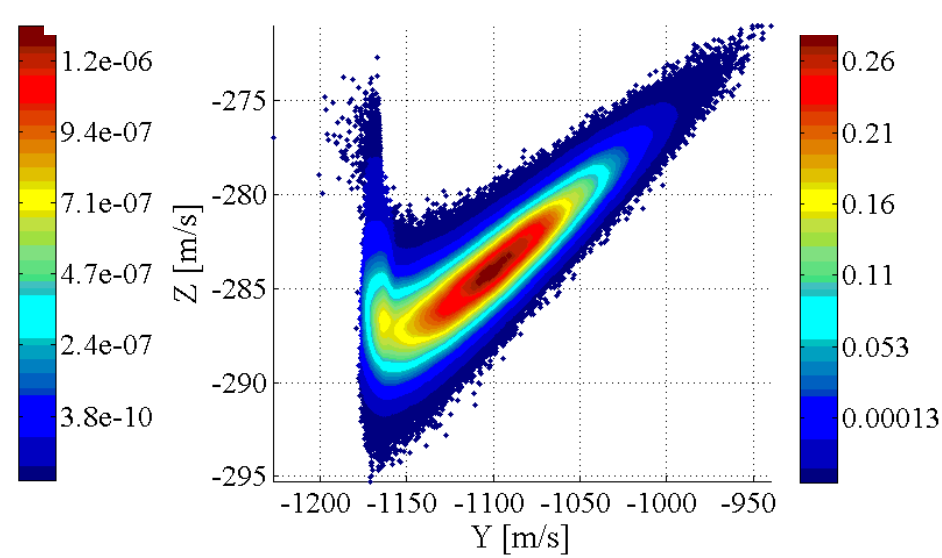

Figure 7. Statistical distribution for Monte Carlo Simulation and 1,000,000 samples: $x-y$ position distribution (a), $\mathrm{x}-\mathrm{z}$ velocity distribution (b). The colorbar represents the probability density.

If we then evaluate the impact rate for these cases as reported in Table 3, we see that the relative difference is below 0.3 per mille between $10^{5}$ and $10^{6}$ samples. We can conclude that $10^{5}$ is a sufficient sample size to describe the distribution of the final states in the case under investigation.

Table 3: Impact rate convergence for increasing number of MC samples.

\begin{tabular}{|c|c|c|c|}
\hline Number of samples & 10,000 & 100,000 & $1,000,000$ \\
\hline Impact rate \% & 19,740 & 19.951 & 20.007 \\
\hline Difference \% & -1.335 & -0.029 & $/$ \\
\hline
\end{tabular}

\subsubsection{Estimation of Mean and Covariance}

The first comparative analysis considers the evolution of the maximum error on the estimation of mean and covariance calculated with the methods in Section 3. The maximum error was defined as the absolute value of the difference between the mean calculated with UT, PCE, SSTs and the one calculated with MCS plus the max norm of the difference between the covariance matrix calculated with UT, PCE, SSTs and the one calculated with MCS. Figure 8 reports the results for the STTs, with order up to 4, and the UT, while Figure 9 shows the same analysis for PCE, with order up to 6. PCE were generated using grids with $l=n$ because we noticed that the best results are achieved when the number of samples is higher than the number of coefficients in the expansion.

Figure 8 shows that the error remains negligible for 40 days till the contribution of the gravity of the Moon becomes significant. At that point the first order STTs depart from the MCS reference solution. At the end of the simulation when the trajectories get closer to the moon and the distribution gets stretched, the order of the STTs needs to be increased to recover the nonlinearities. The accuracy of the results depends on the order for the STTs for both the position (Figure 8a)) and velocity (Figure 8b)). Remarkably, the UT is equivalent to second order STTs at representing the dispersion of the position but is better than a $4^{\text {th }}$ order STT at representing the dispersion of the velocities. Although the UT is not modelling the full shape of the distribution, it is still able to pick a set of points that provides a good approximation of the covariance. The STTs of order 4, instead, do not capture, with sufficient accuracy, the 
deformation of the uncertainty region and as a consequence the following calculation of the covariance results less accurate.

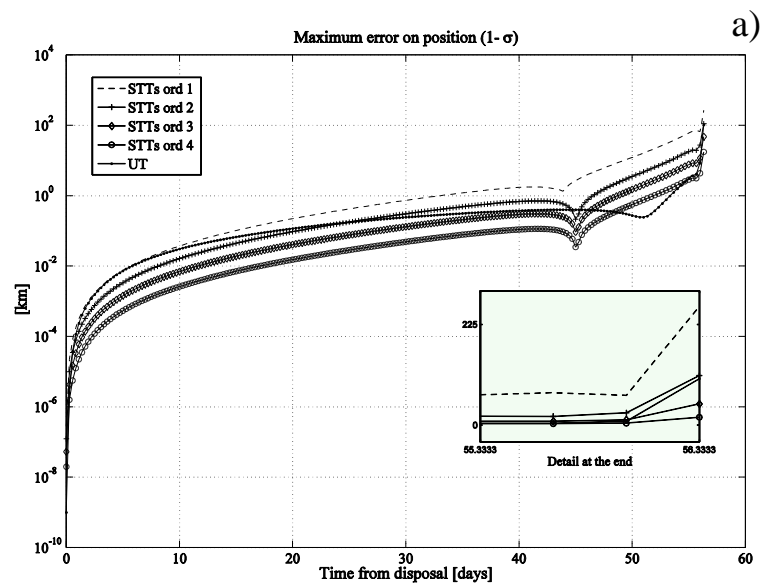

a)

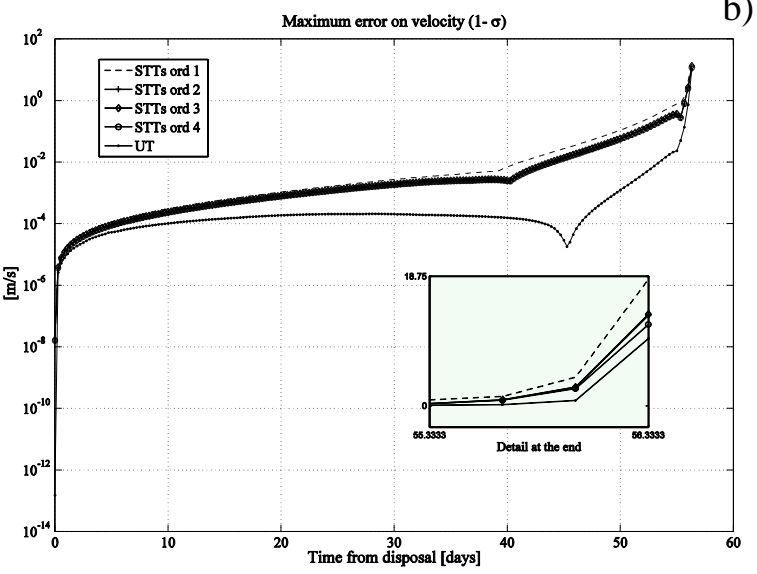

Figure 8. Maximum position (a) and velocity (b) error for STTs and UT with respect to the MC simulation.
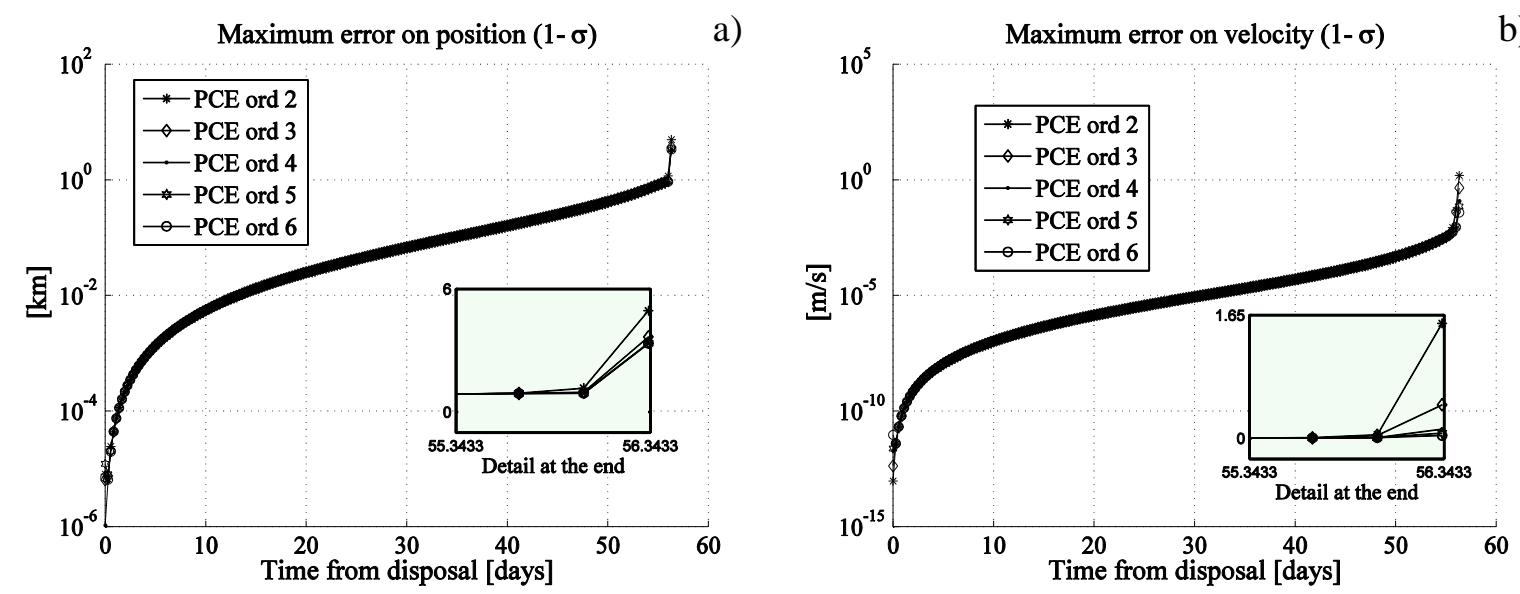

Figure 9. Maximum position (a) and velocity (b) error for STTs and UT with respect to the MC simulation.

The result in Figure 9 shows that, on this test case, a PCE of order 2 achieves a better approximation of mean and variance of an STT of order 4 for both position and velocity. In particular, the error in velocity, at the final time, is about one order of magnitude lower than the $4^{\text {th }}$ order STT and the UT.

\subsubsection{Approximation of the Full Distribution}

The second comparative analysis computes the maximum absolute difference (maximum absolute error) in the distributions of position and velocity at the final time between the STTs, the PCE and the full MCS predictions with $10^{5}$ samples. With the STTs the distribution is derived by integrating all the partials only once and then running a MCS on expansion (16).

Figure 10 to Figure 13 show the resulting distributions for different levels of expansion. Note that the distribution returned by the UT would look like the one in Figure 11. While the position distribution can be approximated quite well with low order STTs (Figure 10a) to Figure 13a)), the same is not possible for the velocity (Figure 10b)) to Figure 13b)). 


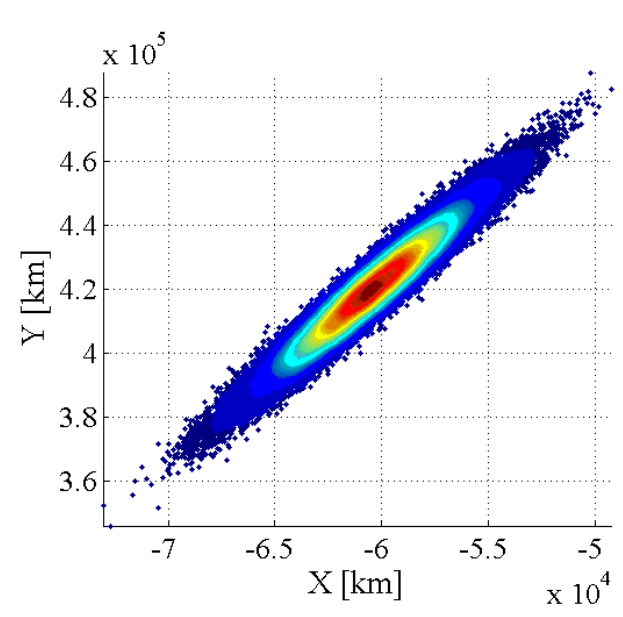

a)

b)

Figure 10. Statistical distribution with $1^{\text {st }}$ order STTs $x-y$ position distribution (a), $x-z$ velocity distribution (b). The colorbar represents the probability density.

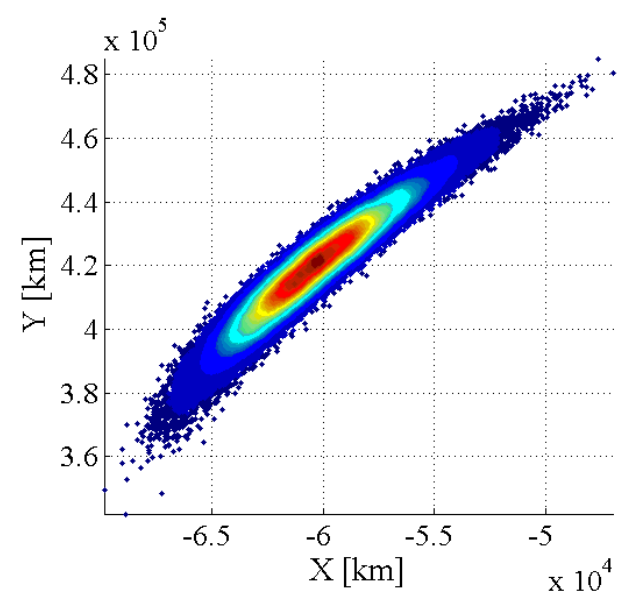

a)
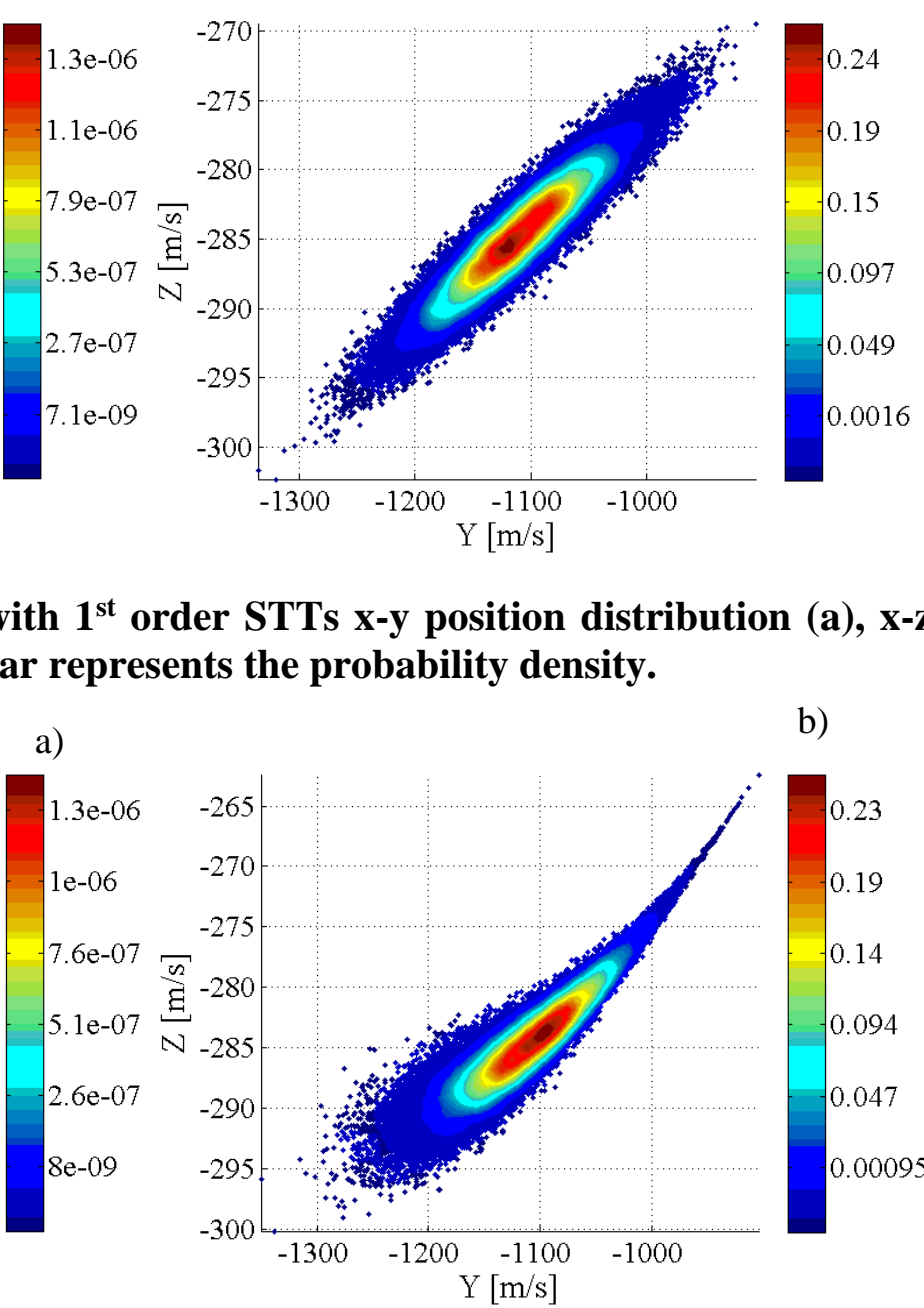

b)

Figure 11. Statistical distribution with $2^{\text {nd }}$ order STTs: $x-y$ position distribution (a), $x-z$ velocity distribution (b). The colorbar represents the probability density.

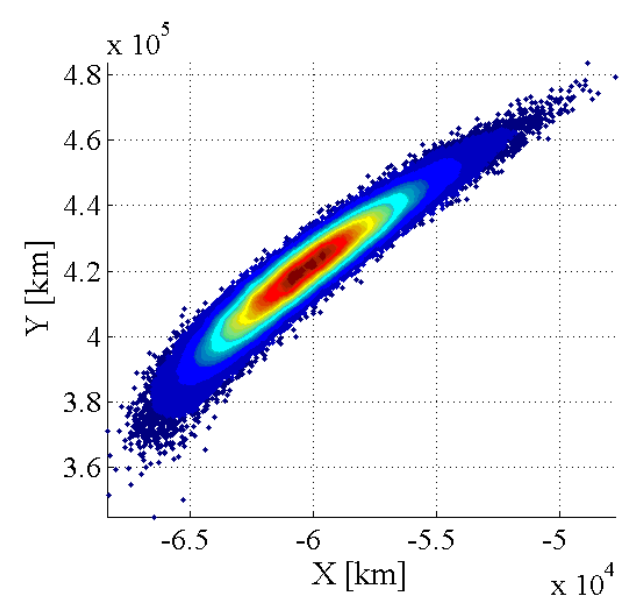

a)
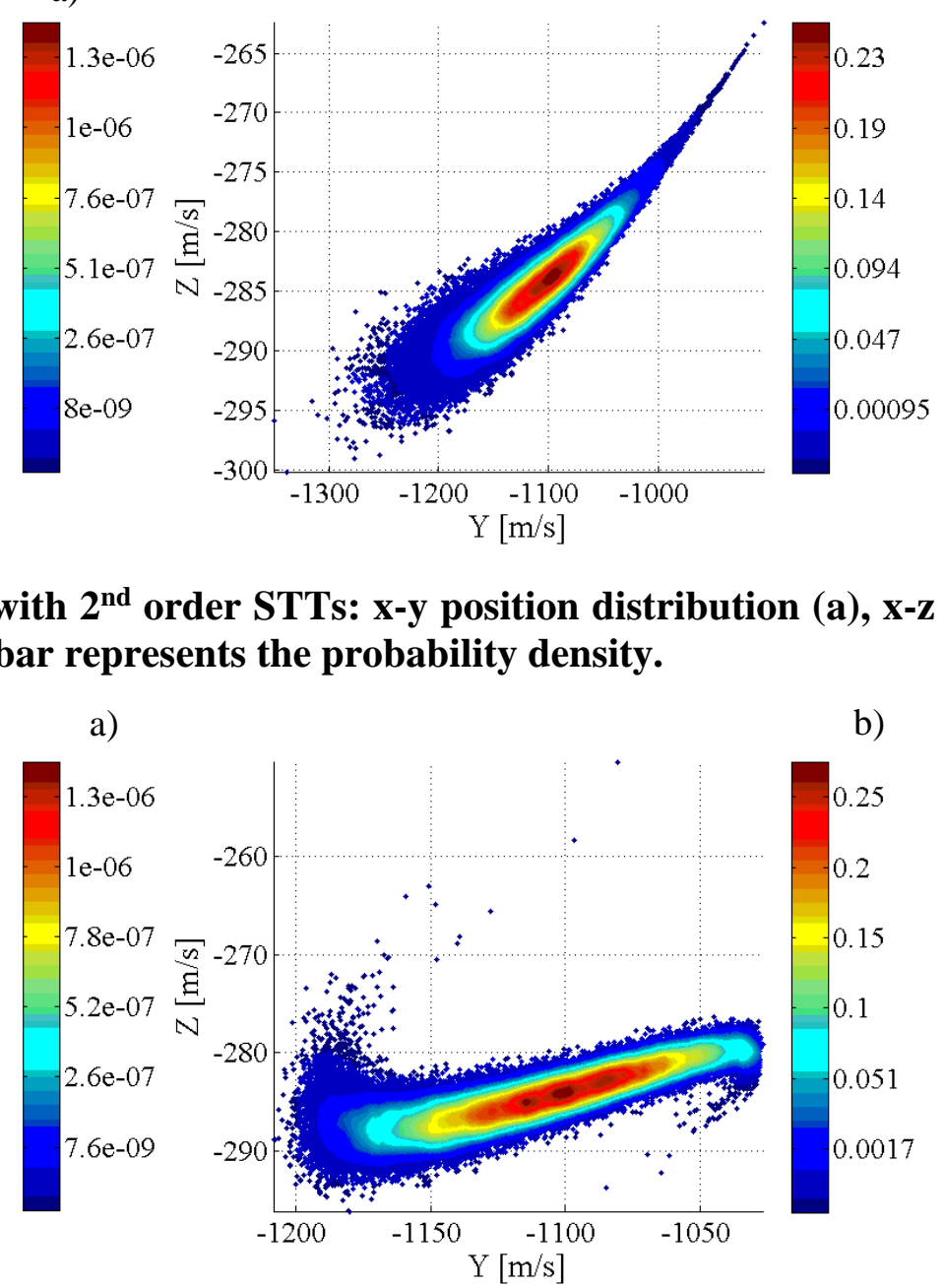

Figure 12. Statistical distribution with $3^{\text {rd }}$ order STTs: $x-y$ position distribution (a), $x-z$ velocity distribution (b). The colorbar represents the probability density. 
a)

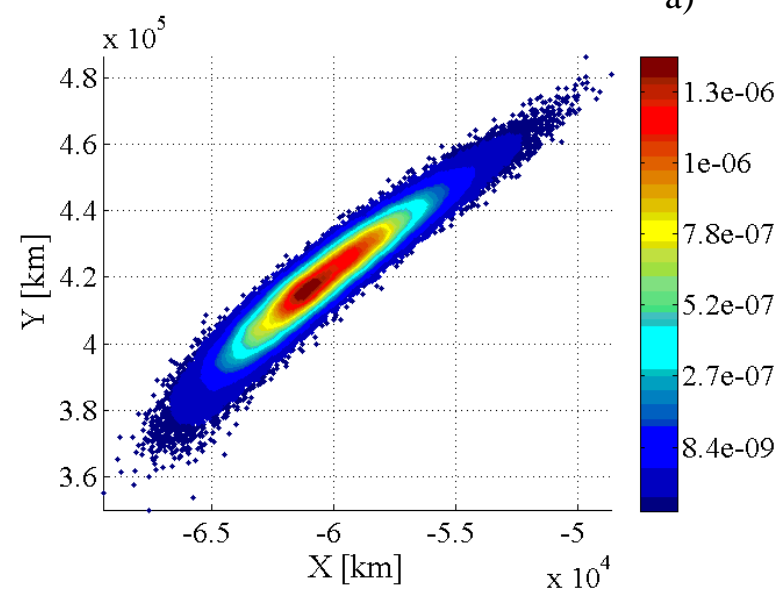

b)

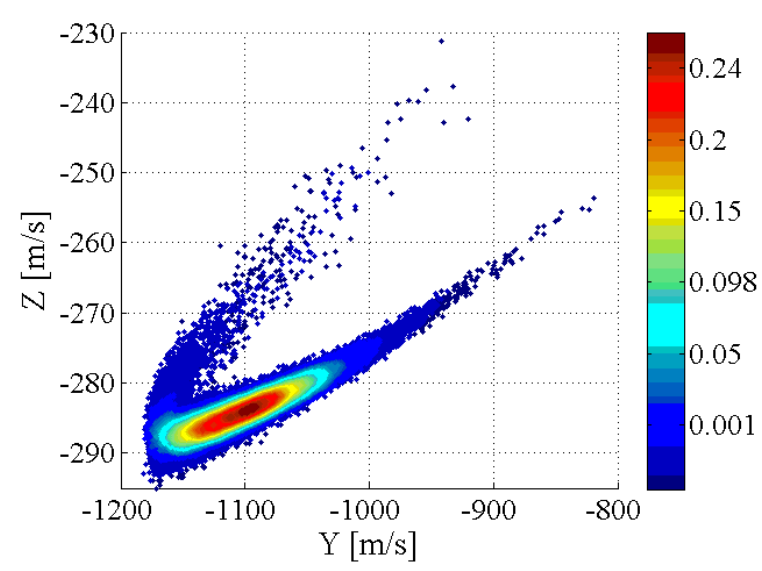

Figure 13. Statistical distribution with $4^{\text {st }}$ order STTs: $x-y$ position distribution (a), $x-z$ velocity distribution (b). The colorbar represents the probability density.

The distribution obtained from STTs of order 1, for both position and velocity, are simply ellipsoids (see Figure 10). This first order approximation partially captures the shape of the distribution in position but fails to represent the velocities. From Figure 11 one can see that the $2^{\text {nd }}$ order STTs distribution of velocity has a teardrop shape which is still far from the result in Figure 7. The third order STT approximation performs slightly better (Figure 12b)) and only the fourth order STTs approximation resembles the uncertain region obtained with the full MCS, although with many samples scattered far from the actual uncertain region (see Figure 13b)).

PCEs show better accuracy in the description of the distribution (see Figure 14 to Figure 18). A $2^{\text {nd }}$ order PCE provides a better representation than $2^{\text {nd }}$ and $3^{\text {rd }}$ order STTs, as can be seen in Figure 14. The representation improves as the order increases from $3^{\text {rd }}$ to $6^{\text {th }}$ (see Figure $15 \mathrm{~b})$ to Figure $18 \mathrm{~b}$ )). The $6^{\text {th }}$ order PCE distributions, in Figure 18, are almost coincident with the full the ones derived from the full MCS. The PCE displayed the remarkable ability to globally represent the distribution better than the SSTs. As to be expected the STTs representation is more accurate nearer the nominal solution and loses accuracy as the sampled points move away from it. The PCE instead is computed by sampling globally over the uncertain space and, thus, achieves a better global representation.
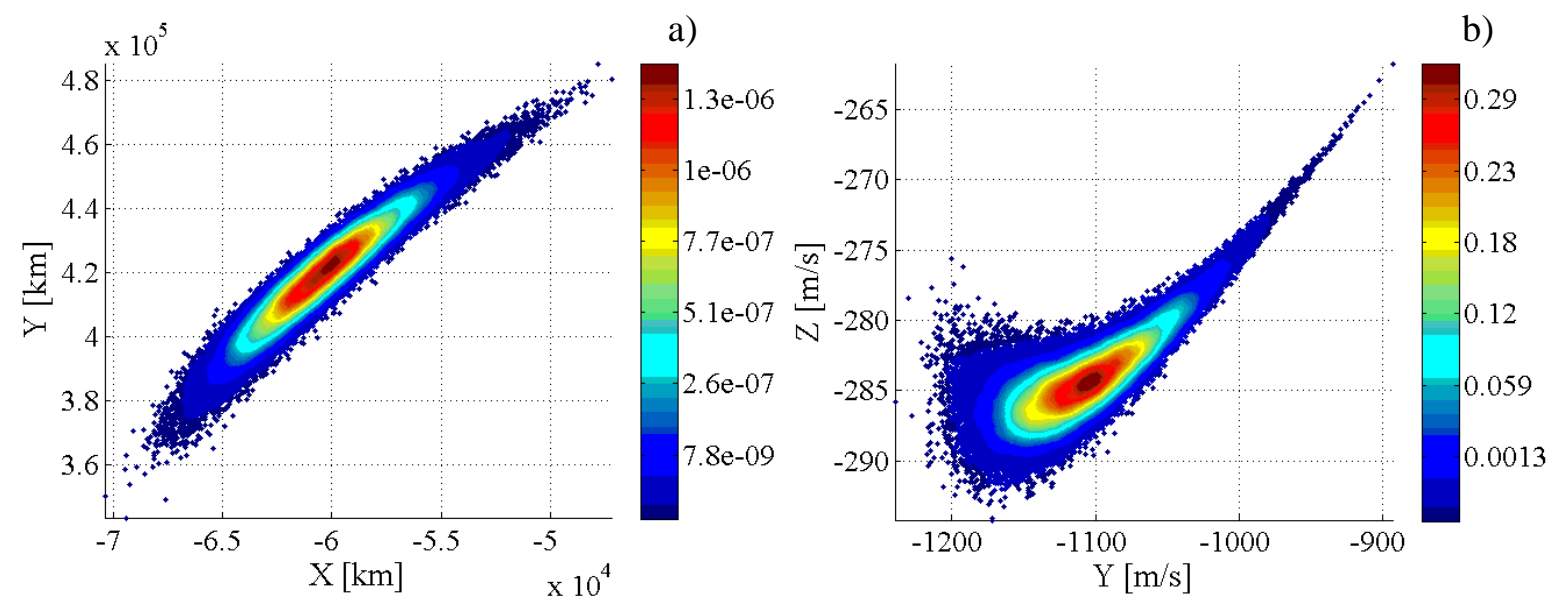

Figure 14. Statistical distribution with $2^{\text {nd }}$ order PCE: $x-y$ position distribution (a), $x-z$ velocity distribution (b). The colorbar represents the probability density. 


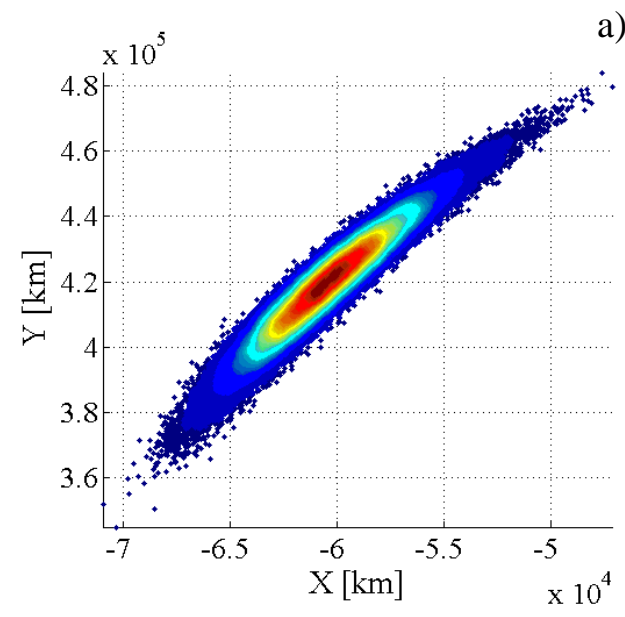

a)
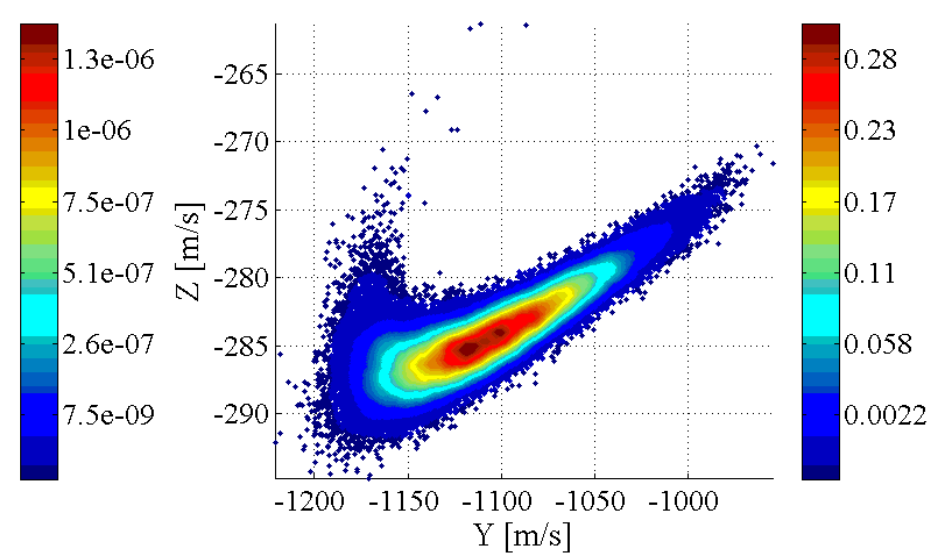

Figure 15. Statistical distribution with 3rd order PCE: $x-y$ position distribution (a), $x-z$ velocity distribution (b). The colorbar represents the probability density.

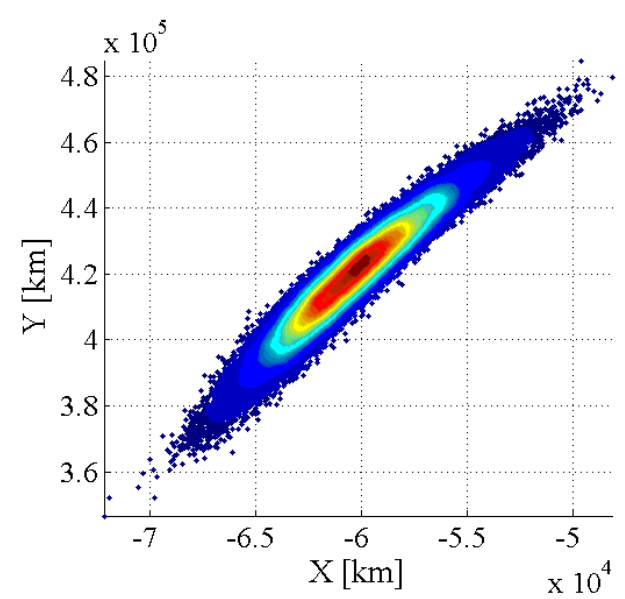

a)

b)
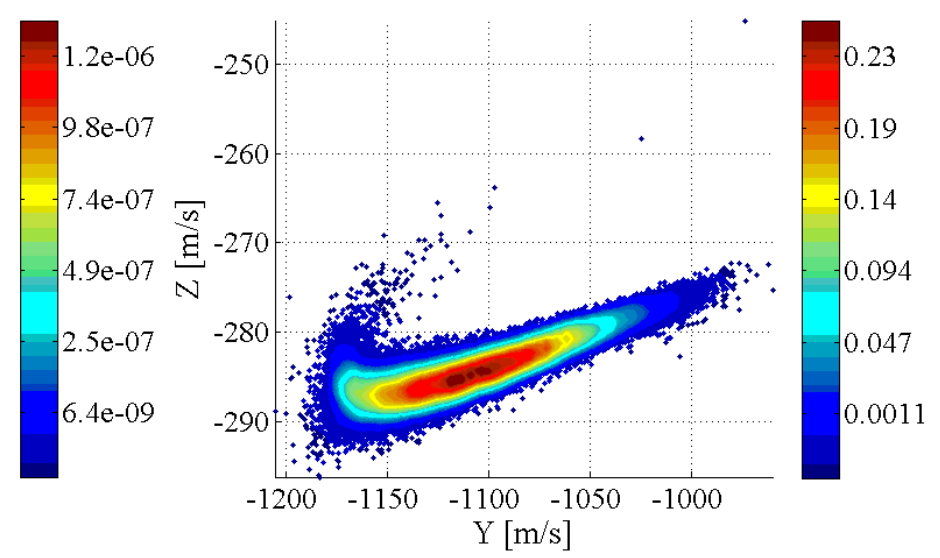

Figure 16. Statistical distribution with $4^{\text {th }}$ order PCE: $x-y$ position distribution (a), $x-z$ velocity distribution (b). The colorbar represents the probability density.

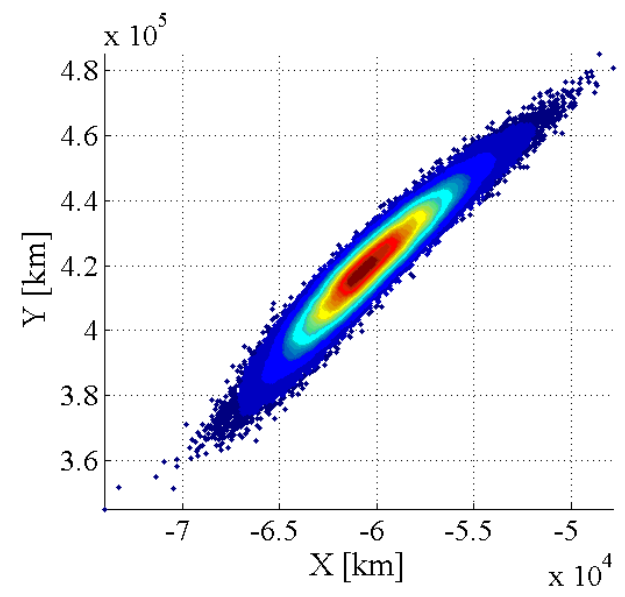

a)
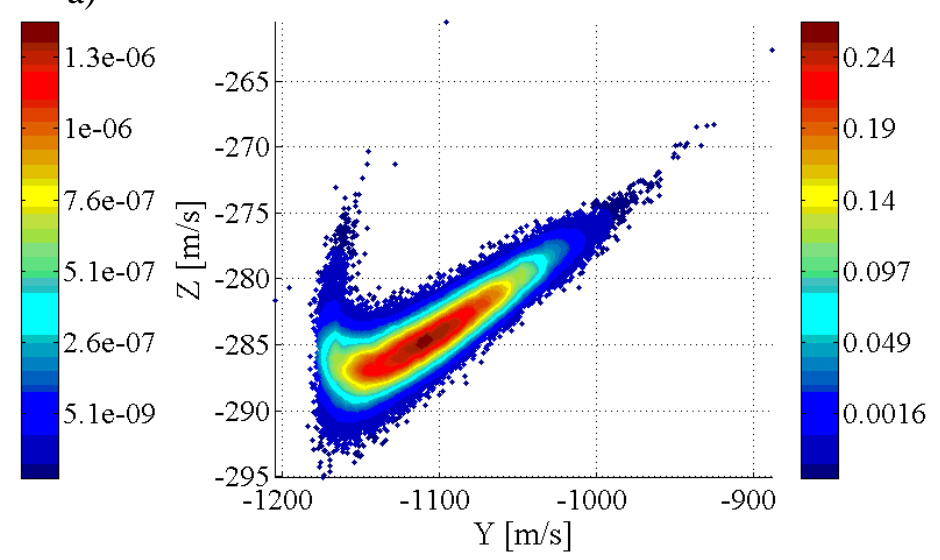

Figure 17. Statistical distribution with $5^{\text {th }}$ order PCE: $x-y$ position distribution (a), $x-z$ velocity distribution (b). The colorbar represents the probability density. 

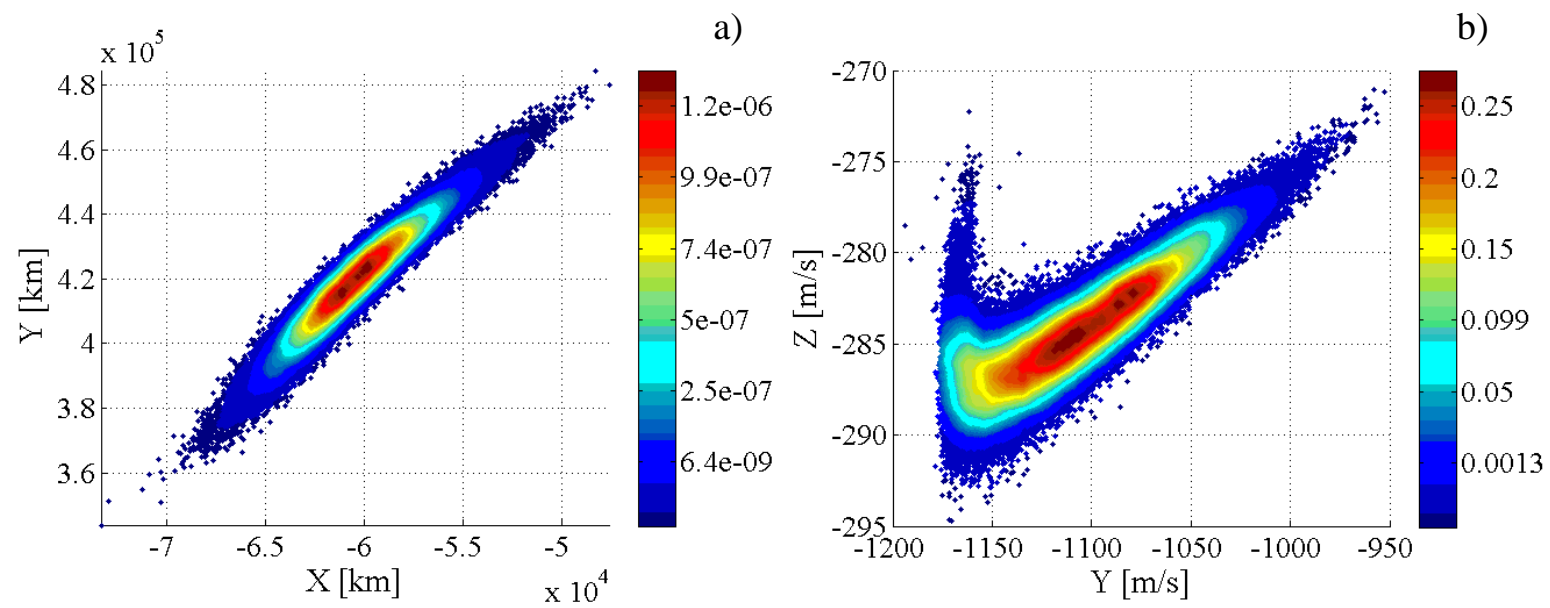

Figure 18. Statistical distribution with $6^{\text {th }}$ order PCE: $x-y$ position distribution (a), $x-z$ velocity distribution (b). The colorbar represents the probability density.

reports the maximum error on the representation of the probability distribution, the number of required integrations, the number of samples, in brackets, and the computational time. In the case of the STT calculation, the number of required integrations is defined as the number of components of the STT that need to be integrated. In all other cases, the number of required integrations is the number of equations to be integrated times the number of samples. For example, for the UT, one needs 13 samples and for each sample 6 differential equations are integrated forward in time. For example, in the case of MCS and PCE the distribution is built by propagating a number of sample points, where each point requires the integration of 6 nonlinear equations.

The computational time, reported in the last column of, includes also the time required to compute the coefficients of the PCE and to resample the STTs and PCE representations. For the PCE expansion the velocity error is, in general, at least one order of magnitude lower than for the STTs and UT. As the order of the PCE increases, the error goes down to less than 2 $\mathrm{cm} / \mathrm{s}$ (for a PCE of order 6). This level of accuracy was obtained with less than one twentieth of the samples of the MCS. The most expensive method is the SST of order 4 as it requires 9,330 integrations of the partials. In fact, the computational cost for a $4^{\text {th }}$ order STTs has the same order of magnitude as the propagation of $10^{5}$ samples of the full MC simulation. A $6^{\text {th }}$ order PCE is as computationally expensive as $3^{\text {rd }}$ order STTs.

The computational cost of the STT can be explained by looking at ratio between the computational time required to evaluate the right hand side of Eqs. (19) to (21) and the time required to evaluate the 6 dynamic equations in (1), times the number of integration steps. In this paper all partials required to compute the STT were generated with a symbolic manipulator and the full analytical expressions were inserted in a Matlab function. All calculations were vectorised to improve speed. As an example, we considered the propagation of a single trajectory for 0.25 days using the nominal initial conditions specified in section 3.3. For the single trajectory the propagator required 18 time steps. The propagation of a $1^{\text {st }}$ order STT required 28 integration steps and 7.35 times the computational cost of a single integration of the dynamics. The propagation of a $2^{\text {nd }}$ order STT required 30 integration steps and about 51.62 times the computational cost of a single integration of the dynamics. Likewise the propagations of a $3^{\text {rd }}$ and $4^{\text {th }}$ order STTs required 35 integration steps and resulted to be, respectively, 833.14 and 17,710 more expensive than a single integration of the dynamics. An STT of order 3 is, therefore, almost as expensive as a PCE of order 4 
though delivering a significantly worse approximation of the distribution. To be noted, however, that the cost of the STTs can be further reduced by exploiting the symmetries and (potential) sparsity in their structure, as well as ad hoc propagation schemes. Symmetry and sparsity are problem dependent and were not exploited in these simulations.

Table 4: Comparison between STTs, PCE and MCS for a 56 days propagation from LPO to Moon disposal manoeuvre. A 64-bit Linux CentOS $5.42 .93 \mathrm{GHz}$ Intel $^{(\mathrm{R})}$ Xeon $^{(\mathrm{TM})}$ X5570 was used.

\begin{tabular}{|l|l|l|l|l|}
\hline Method & $\begin{array}{l}\text { Maximum position } \\
\text { error }(1-\sigma)[\mathrm{km}]\end{array}$ & $\begin{array}{l}\text { Maximum of } \\
\text { velocity error } \\
(1-\sigma)[\mathrm{m} / \mathrm{s}]\end{array}$ & $\begin{array}{l}\text { Number } \\
\text { integrations } \\
\text { (samples })\end{array}$ & CPU time [s] \\
\hline UT & 107.594 & 8.869 & $78(13)$ & 63.53 \\
\hline STTs $n=1$ & 265.196 & 18.282 & 42 & 22 \\
\hline STTs $n=2$ & 110.323 & 13.061 & 258 & 154 \\
\hline STTs $n=3$ & 47.295 & 13.222 & 1,554 & 2,492 \\
\hline STTs $n=4$ & 17.470 & 11.743 & 9,330 & 52,970 \\
\hline PCE $n=2$ & 4.936 & 1.539 & $438(73)$ & 222 \\
\hline PCE $n=3$ & 3.660 & 0.449 & $1,542(257)$ & 773 \\
\hline PCE $n=4$ & 3.356 & 0.122 & $4,494(749)$ & 2,244 \\
\hline PCE $n=5$ & 3.353 & 0.070 & $12,126(2021)$ & 6,050 \\
\hline PCE $n=6$ & 3.344 & 0.038 & $28,350(4725)$ & 14,139 \\
\hline MCS & 0 & 0 & $\begin{array}{l}600,000 \\
(100,000)\end{array}$ & 299,093 \\
\hline
\end{tabular}

For the calculations presented in the previous paragraph and in Table 4 we considered an adaptive step size integrator of Section 4.1. Thus, part of the cost of the STT, compared to a single integration of the dynamics, comes from the increased number of steps. Therefore, in order to remove any bias introduced by the integrator we propagated all STTs with a RungeKutta integrator of order 5 with a fixed step size of about 0.3 days (i.e. one two-hundredth of the disposal interval). The result, reported in Table 5, shows an improvement in the computational time but a worsening of the accuracy. For order four, the position error is 20 times bigger than in the case of the variable step-size integrator.

Table 5: STTs performance using a fixed step Runge-Kutta integrator of order 5.

\begin{tabular}{|l|l|l|l|}
\hline Method & $\begin{array}{l}\text { Maximum position } \\
\text { error }(1-\sigma)[\mathrm{km}]\end{array}$ & $\begin{array}{l}\text { Maximum } \\
\text { velocity error } \\
(1-\sigma)[\mathrm{m} / \mathrm{s}]\end{array}$ & CPU time [s] \\
\hline STTs $n=1$ & $1,711.33$ & 32.29 & 20.7 \\
\hline
\end{tabular}




\begin{tabular}{|l|l|l|l|}
\hline STTs $n=2$ & $1,643.66$ & 29.70 & 137.8 \\
\hline STTs $n=3$ & 686.08 & 24.83 & 1,981 \\
\hline STTs $n=4$ & 558.28 & 23.99 & 33,960 \\
\hline
\end{tabular}

\subsection{Impact Probability Analysis}

The analyses in Section 4.1.2 and 4.1.3, have shown that, in the case under investigation, PCEs offer a better approximation of the final distribution compared to UT and STTs and a lower computational cost compared to STTs and MCS. Therefore, in this section, PCEs are the method of choice to estimate the impact rate with the Moon. The distribution of virtual impactors is reconstructed 8 hours before the time of the nominal impact. Then for the $i$-th virtual impactor the osculating orbital elements of an orbit intersecting the Moon are computed and the anomaly corresponding to the intersection with the surface of the Moon $\theta_{\text {impact }}$, is obtained simply from:

$$
r\left(\theta_{\text {impact }}, \boldsymbol{\chi}_{\mathrm{i}}\right)=R_{\text {Moon }}
$$

The approximation in Eq. (35) allows one to use a lower order representation of the distribution at the Moon and avoid the discontinuity due to the fact that some virtual impactors hit the surface and others miss the Moon. The Cartesian coordinates at impact are then recovered from $\theta_{\text {impact }}$ and the orbital elements of the virtual impactor. For these analyses we used the full dynamical model.

Table 6 shows the impact rate for MCS and PCE using $10^{5}$ virtual impactors. From this table one can see that the difference between PCE and MCS progressively reduces as the order of the PCE increases. The increase in the error between order 2 and 3 is probably due to numerical inaccuracies in the calculation of the coefficients of the PCE expansion given the extended uncertainty region. In fact, as shown below in the case with a reduced uncertainty, order 3 is more accurate than order 2.

Table 6: Impact rate comparison between MCS and PCE: 1\% manoeuvre error and 5 km dispersion error (per component).

\begin{tabular}{|c|c|c|c|c|c|c|}
\hline & MCS & PCE ord 2 & PCE ord 3 & PCE ord 4 & PCE ord 5 & PCE ord 6 \\
\hline Impact rate \% & 19.951 & 20.858 & 21.007 & 20.515 & 20.430 & 20.180 \\
\hline Relative error \% & $/$ & 4.55 & 5.41 & 2.83 & 2.40 & 1.14 \\
\hline
\end{tabular}

The small error in the impact rate is consistent with the small error in the distribution of the virtual impactor. Figure 19 to Figure 21 report the density and distribution of the virtual impactors on the south hemisphere of the Moon. The density distribution was calculated as the percentage of impactors hitting a circle of $30 \mathrm{~km}$ radius centred on each virtual impactor divided by the corresponding area.

For order 2 the PCE representation is particularly effective at reconstructing the area closer to the mean, given the shape of the grid shown in Figure 4 of Section 3.4.1, with all virtual impactors concentrated in the area closer to the nominal trajectory. The MCS distribution, however, appears to be multimodal, which is better captured by a higher order PCE. To be 
noted that given the low impact rate, these considerations are limited to the distribution only of the virtual impactors that hit the Moon, while Table 6 accounts also for the virtual impactors that miss the Moon. As one can see, MCS and PCE return a similar distribution and density of virtual impactors already for order 2 with a progressive improvement as the order increases.

a)

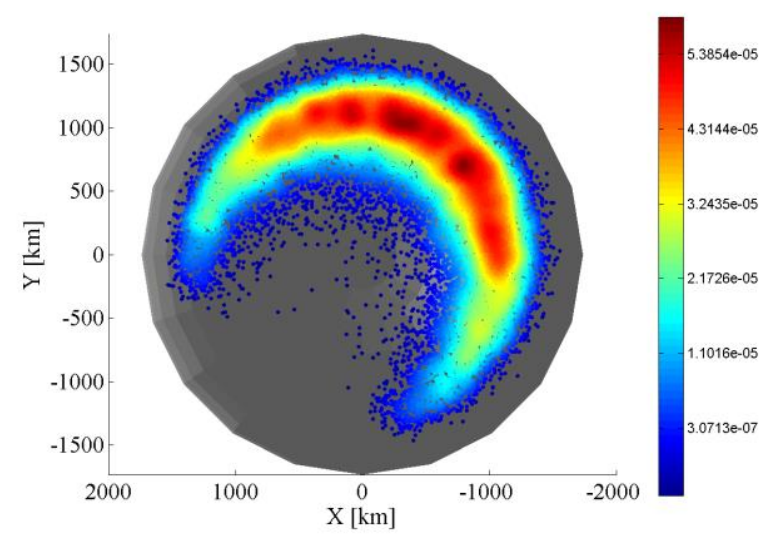

b)

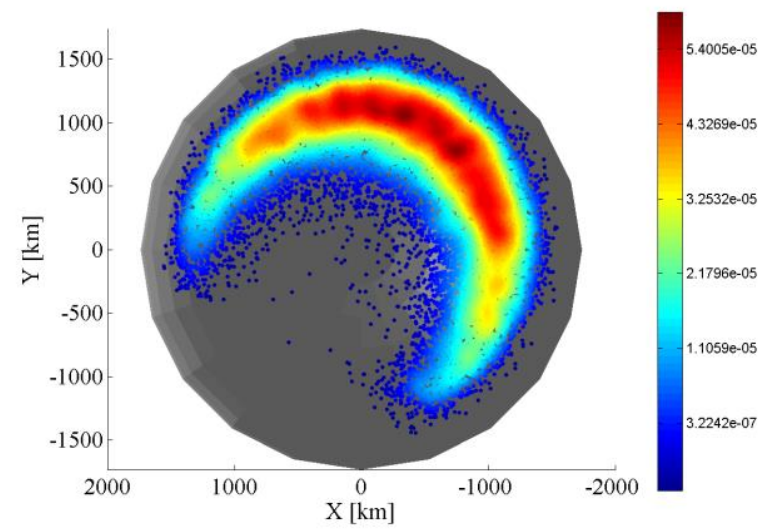

Figure 19. Particles dispersion and density at impact for $1 \%$ manoeuvre error and $5 \mathrm{~km}$ dispersion error (per component): MCS (a) and PCE order 2 (b). The colorbar represents the probability density.
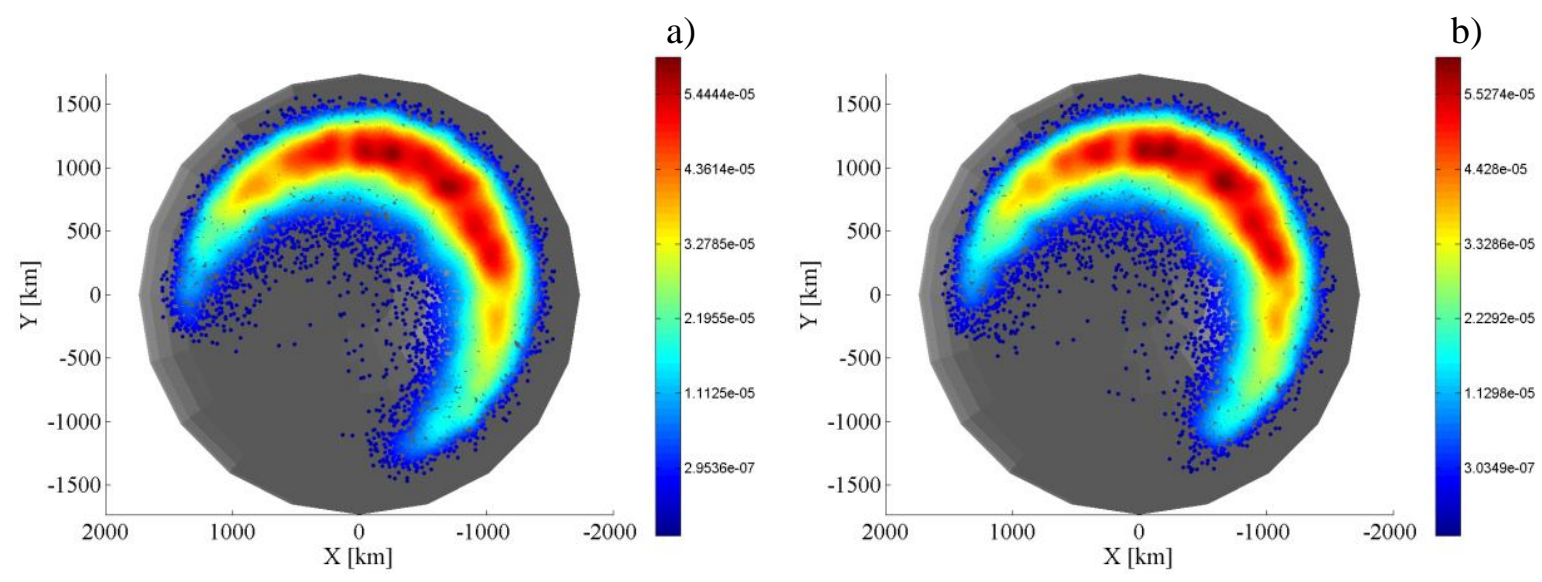

Figure 20. Particles dispersion and density at impact for $1 \%$ manoeuvre error and $5 \mathrm{~km}$ dispersion error (per component): PCE order 3 (a) and PCE order 4 (b). The colorbar represents the probability density. 
a)

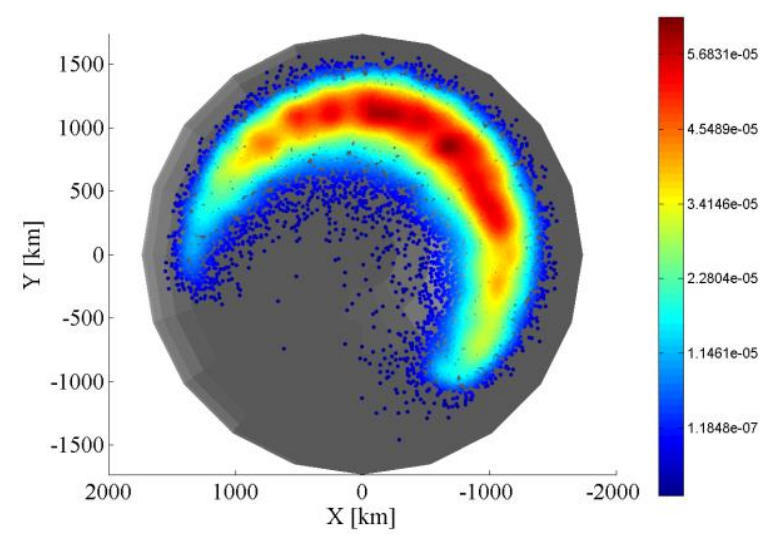

b)

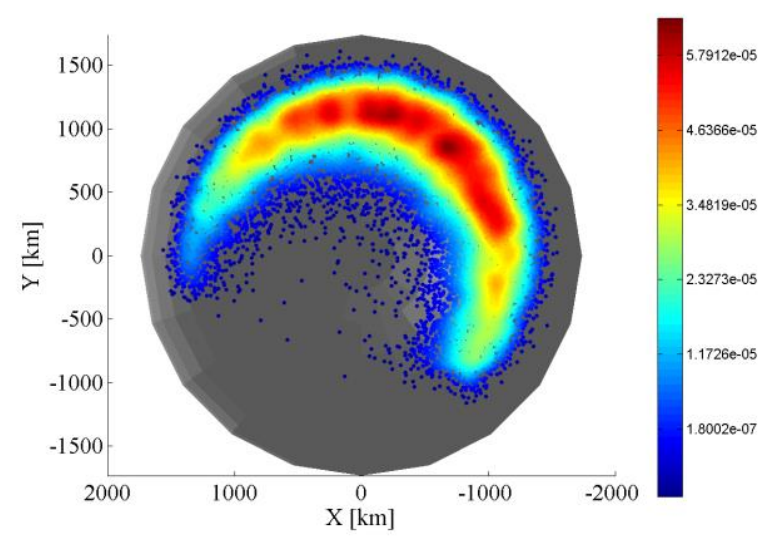

Figure 21. Particles dispersion and density at impact for $1 \%$ manoeuvre error and $5 \mathrm{~km}$ dispersion error (per component): PCE order 5 (a) and PCE order 6 (b).

One can now check the sensitivity to a resampling of the PCE with a higher number of virtual impactors. Figure 21 shows the result for 200,000 samples and an order 6 PCE. The resulting distribution appears even closer to the MCS one with comparable density of virtual impactors.

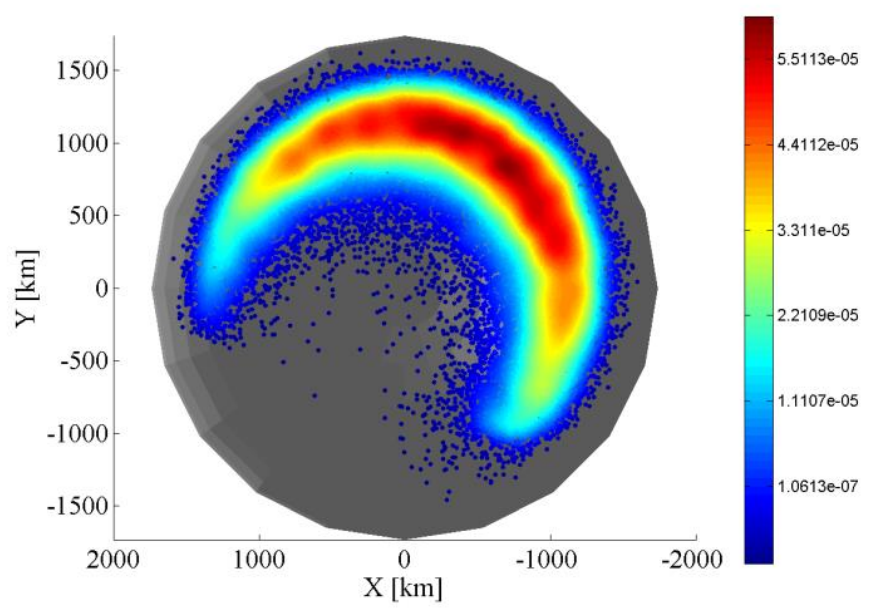

Figure 22. Particles dispersion and density at impact for $1 \%$ manoeuvre error and $5 \mathbf{~ k m}$ dispersion error (per component): PCE order 6 with 200,000 samples. The colorbar represents the probability density.

The previous example demonstrated that a $1 \%$ manoeuvre error leads to a low impact probability. If an impact probability higher than $90 \%$ has to be guaranteed a lower error in orbit determination and manoeuvre execution is required. One can now consider the case where the dispersion in position is limited to $1 \mathrm{~km}$ per component, while the one for the velocity is just $0.1 \%$ of the disposal manoeuvre. Table 7 shows the resulting impact probability of both MCS and PCE. As in the previous case, one can see that the higher order PCE are more accurate in the description of the overall impact statistics. The difference between MCS and PCE is only $0.03 \%$ for an order 2 PCE and drops to $0.005 \%$ for a $6^{\text {th }}$ order PCE.

Table 7. Impact rate comparison between MCS and PCE: 0.1\% manoeuvre error and 1 km dispersion error (per component). 


\begin{tabular}{|c|c|c|c|c|c|c|}
\hline & MCS & PCE ord 2 & PCE ord 3 & PCE ord 4 & PCE ord 5 & PCE ord 6 \\
\hline Impact rate \% & 92.326 & 92.354 & 92.337 & 92.331 & 92.331 & 92.329 \\
\hline Relative error \% & $/$ & 0.03 & 0.012 & 0.005 & 0.005 & 0.004 \\
\hline
\end{tabular}

As before, we show also the corresponding distribution and probability density of the virtual impactors. From Figure 23 to Figure 25 one can see that the virtual impactors are less scattered with respect to the previous case. Although the distribution is stretched over a half circle, the probability density function appears unimodal with the maximum density that is approximately one order of magnitude higher than in the previous case $\left(4.5 \cdot 10^{-4}\right.$ against $\left.5.4 \cdot 10^{-5}\right)$.

a)
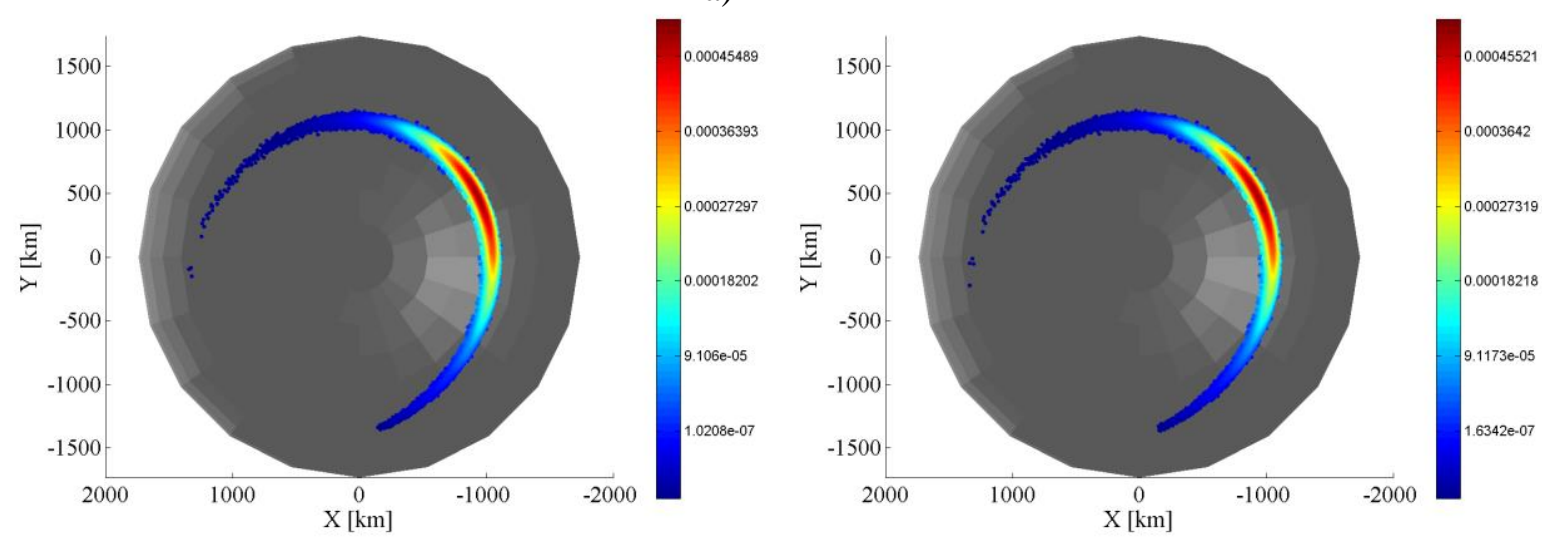

Figure 23. Particles dispersion and density at impact for $0.1 \%$ manoeuvre error and 1 km dispersion error (per component): MCS (a) and PCE order 2 (b). The colorbar represents the probability density.

a)

b)
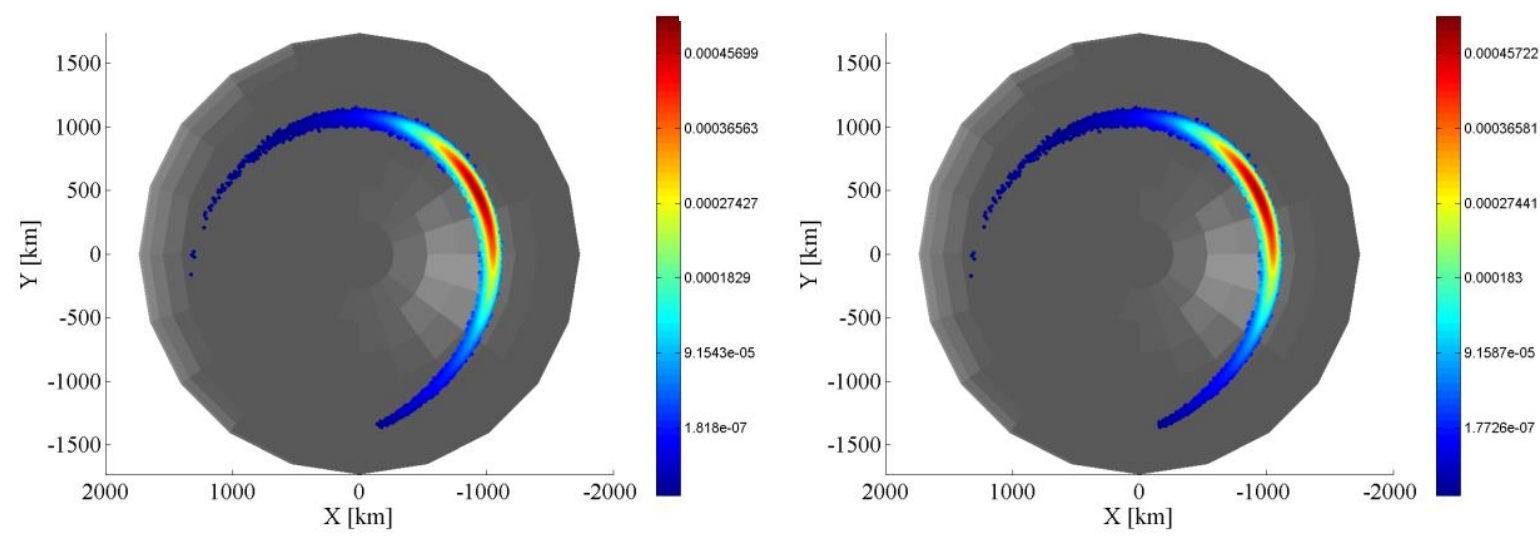

Figure 24. Particles dispersion and density at impact for $0.1 \%$ manoeuvre error and 1 km dispersion error (per component): PCE order 3 (a) and PCE order 4 (b). The colorbar represents the probability density. 
a)

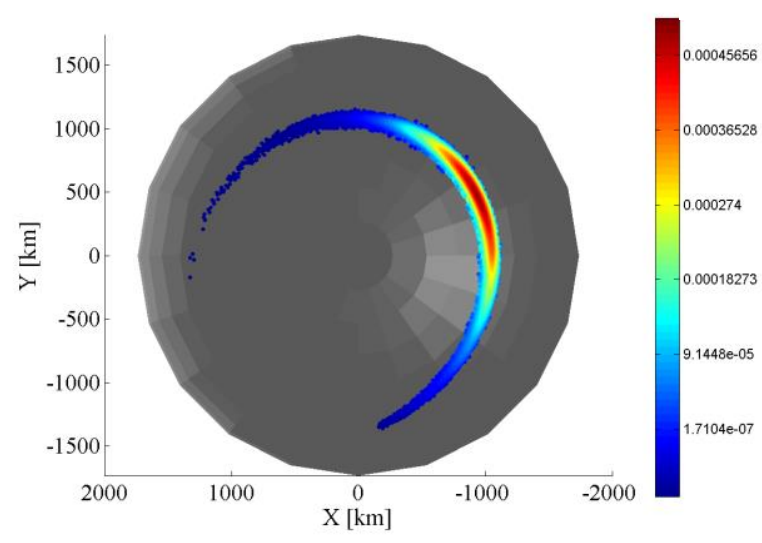

b)

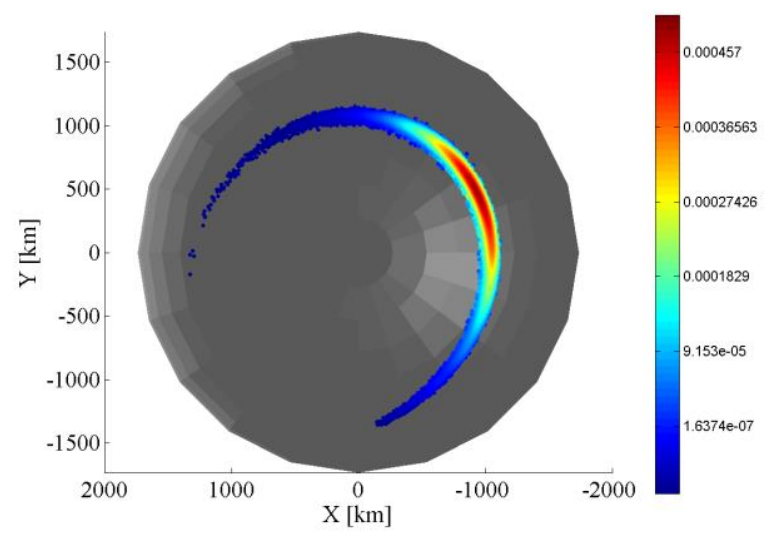

Figure 25. Particles dispersion and density at impact for $0.1 \%$ manoeuvre error and 1 km dispersion error (per component): PCE order 5 (a) and PCE order 6 (b).

\section{Conclusions}

In this paper we have applied different techniques for Uncertainty Quantification to the study of the disposal from LPO to the Moon. We studied in particular the disposal of Herschel from the Lagrangian Point L2 where the initial disposal conditions are affected by uncertainties due to both orbit determination and system performance.

From this study it emerged that PCE are an appropriate tool for this type of analyses and provide accurate predictions at low computational cost. The particular PCE proposed in this paper is based on multivariate Hermite polynomials with integration over Genz and Keister sparse-grids. Multivariate Hermite polynomials were chosen because they have infinite support and can well represent mixtures of Gaussian distributions. Two key features of PCE were exploited in this paper: the ability to produce functional representations of any generic stochastic distribution and the direct derivation of the statistical moments from the coefficients of the PCE expansion. The latter was used when comparing the mean and the covariance against UT and low order SSTs, while the former was exploited when computing the impact rate.

In the case investigated in this paper, Taylor expansions based on the numerical propagation of STTs have proved to be not as efficient and accurate as PCEs. This is a problem when several reference trajectories have to be tested. On the contrary the PCE expansion proved to be a valid alternative to MCS, featuring less computational effort and comparable accuracy.

The impact probability analysis provided an understanding of the effectiveness of the disposal manoeuvre in relation to the accuracy with which the initial position and velocity pre manoeuvre are known and the accuracy with which the disposal manoeuvre is performed. In order to achieve over $90 \%$ success rate, the initial accuracy on the disposal manoeuvre has to be as low as $0.1 \%$ of the nominal value with $1 \mathrm{~km}$ dispersion in position. A $1 \%$ error in the manoeuvre and $5 \mathrm{~km}$ of uncertainty in position reduces the impact success rate to only $20 \%$.

Future work will see the application of PCE to the study of uncertainties effects on other disposal trajectories which can involve multi-revolutions around the Moon and the Earth and fly-bys and the treatment of singularities or mixed regimes of motion (for example atmospheric entry combined with orbital regime) that might require a partitioning of the uncertain domain. 


\section{Acknowledgements}

The work is partially supported by the Marie Curie FP7-PEOPLE-2012-ITN Stardust, grant agreement 317185. Results were obtained using the EPSRC funded ARCHIE-WeSt High Performance Computer (www.archie-west.ac.uk). EPSRC grant no. EP/K000586/1.

\section{References}

1. Agarwal, N., Aluru, N. R.: Weighted Smolyak algorithm for solution of stochastic differential equations on non-uniform probability measures. International Journal for Numerical Methods in Engineering, Int. J. Numer. Meth. Engng 2011, Vol. 85, pp. $1365-$ 1389. DOI: $10.1002 / \mathrm{nme} .3019$.

2. Babuska I, Nobile F, Tempone R.: A stochastic collocation method for elliptic partial differential equations with random input data. SIAM Journal on Numerical Analysis 2007; 45(3):1005-1034. DOI:10.1137/050645142.

3. Blanchard, E. D., Sandu, A., and Sandu, C.: A Polynomial Chaos-Based Kalman Filter Approach for Parameter Estimation of Mechanical Systems, ASME Journal of Dynamic Systems, Measurement, and Control, 2010;132(6):061404-061404-18. doi: $10.1115 / 1.4002481$

4. Jones B., A., and Alireza, D.: Satellite Collision Probability Estimation Using Polynomial Chaos. Advances in Space Research Volume 52, Issue 11, 1 December 2013, Pages 1860-1875. http://dx.doi.org/10.2514/1.53793

5. Cheng, Y., Jia, B. and Xin, M.: Sparse Grid Approach to Orbit Uncertainty Propagation. in ICCES: International Conference on Computational \& Experimental Engineering and Sciences, vol. 16, no. 2, pp. 33-34, 2011. doi:10.3970/icces.2011.016.033

6. Colombo, C., Alessi, E.M., van der Weg, W., Soldini, S., Letizia, F., Vetrisano, M., Vasile, M. Rossi, A., Landgraf, M.: End-of-life disposal concepts for Libration Point Orbit and Highly Elliptical Orbit Missions, Acta Astronautica, Volume 110, May-June 2015, Pages 298-312, Dynamics and Control of Space Systems. doi:10.1016/j.actaastro.2014.11.002

7. Colombo, C., Alessi, E.M., van der Weg, W., Soldini, S., Letizia, F., Vetrisano, M., Vasile, M. Rossi, A.: End-of-life disposal concepts for Libration Point Orbit and Highly Elliptical Orbit Missions, Executive summary of the main study and the study extension, Version 1.0 - 12 February 2015 ESA/ESOC contract No. 4000107624/13/F/MOS

8. Eldred M. S., Swiler L.P., and Tang, G.: Mixed aleatory-epistemic Uncertainty quantification with stochastic expansions and optimization-based interval estimation. Reliability Engineering and System Safety, Vol. 96, 2011, pp. 1092-1113. doi:10.1016/j.ress.2010.11.010

9. Ganapathysubramanian B, Zabaras N.: Sparse grid collocation schemes for stochastic natural convection problems. Journal of Computational Physics 2007; 225:652-685. doi:10.1016/j.jcp.2006.12.014

10. Genz, A. and Keister, B.: Fully Symmetric Interpolatory Rules for Multiple Integrals over Infinite Regions with Gaussian Weight, J. Comp. Appl. Math. 71 (1996), pp. 299-309. doi:10.1016/0377-0427(95)00232-4

11. Ghanem R.G. and Spanos P.: Stochastic Finite Elements: A Spectral Approach. Springer: Berlin, 1991. doi:10.1007/BF02818931

12. Ghanem, R. and Dham, S.: Stochastic finite element analysis for multiphase flow in heterogeneous porous media. Transporting Porous Media, Vol. 32, No. 3, pp. 239262, 1998. doi:10.1023/A:1006514109327 
13. Ghanem R., Doostan A.: On the construction and analysis of stochastic models: characterization and propagation of the errors associated with limited data. Journal of Computational Physics 2006; 217:63-81. http://dx.doi.org/10.1016/j.jcp.2006.01.037

14. Gilli, L., Lathouwers, D., Kloosterman, J.L., and van der Hagen, T.H.J.J.: Performing uncertainty analysis of a nonlinear Point-Kinetics/Lumped Parameters problem using Polynomial Chaos techniques. Annals of Nuclear Energy 40 (2012) 35-44. DOI: 10.1016/j.anucene.2011.09.016

15. Godard, B., Croon, M., Budnik, F. and Morley, T.: Orbit determination of the Plank Satellite. The $21^{\text {st }}$ International Symposium on Space Flight Dynamics. Toulouse, France, 2009.

16. Heiss F. and Winschel V.: Likelihood approximation by numerical integration on sparse grids, Journal of Econometrics (2008), doi:10.1016/j.jeconom.2007.12.004

17. Hosder, S., Walters, R. W., and Perez, R.: A Non-Intrusive Polynomial Chaos Method for Uncertainty Propagation in CFD Simulations. 44th AIAA Aerospace Sciences Meeting and Exhibit, Reno, Nevada, January 9 - 122006.

18. Jones, B. A., Doostan, A. and Born, G. H.: Nonlinear Propagation of Orbit Uncertainty Using Non-Intrusive Polynomial Chaos. Journal of Guidance, Control, and Dynamics, Vol. 36, No. 2 (2013), pp. 430-444. DOI:10.2514/1.57599

19. Julier, J. K. Uhlmann and Durrant-Whyte, H.F.: A new approach for filtering nonlinear systems, Proceedings of the American Control conference, Seattle, Washington, 1995. DOI: 10.1109/ACC.1995.529783

20. Knio, O. M. and Le Maître, O. P. L.: Uncertainty Propagation in CFD Using Polynomial Chaos Decomposition. Fluid Dynamics Research, Vol. 38, No. 9, September 2006, pp. 616-640. doi:10.1016/j.fluiddyn.2005.12.003

21. Kubota, T., Hashimoto, T. et al.: An autonomous navigation and guidance system for MUSES-C asteroid landing. Acta Astronautica, 2003, 52(2-6): 125-131.

http://dx.doi.org/10.1016/S0094-5765(02)00147-9

22. Le Mâitre O.P., Ghanem R.G., Knio, O.M., Najm H.N.: Uncertainty propagation using Wiener-Haar expansions. Journal of Computational Physics 2004; 197:28-57. http://dx.doi.org/10.1016/j.jcp.2003.11.033

23. Lewis, L., and Parkinson, A.: Robust Optimal Design Using a Second Order Tolerance Model, Research in Engineering Design, Vol. 6, No. 1, 1994, pp. 25-37. doi:10.1007/BF01588089

24. Mardia, K. V., Kent, J. T. and Bibby, J. M.: 'Multivariate Analysis'. Academic Press, London, 1979. DOI: 10.1002/bimj.4710240520

25. Najm, H. N.: Uncertainty Quantification and Polynomial Chaos Techniques in Computational Fluid Dynamics. Annual Reviews of Fluid Mechanics, Vol. 41, 2009, pp. 35-52. DOI: 10.1146/annurev.fluid.010908.165248

26. Padulo, M., Campobasso, M. S. and Guenov M. D.: A novel Uncertainty Propagation Method for Robust Aerodynamic Design. AIAA Journal, 49(3):530-543, 2011. doi:10.2514/1.J050448

27. Parkinson, A., Sorensen, C., and Pourhassan,: A General Approach for Robust Optimal Design, Journal of Mechanical Design, Vol. 115, No. 1, 1993, pp. 74-80. doi:10.1115/1.2919328

28. Park, R. and Scheeres, D.: Nonlinear Semi-Analytic Method for Spacecraft Navigation, AIAA/AAS Astrodynamics Specialist Conference and Exhibit, Keystone, Colorado, 2124 August 2006.

29. Riccardi, A., Tardioli, C., Vasile, M., An Intrusive Approach to Uncertainty Propagation in Orbital Mechanics Based on Tchebycheff Polynomial Algebra. Astrodynamics Specialists Conference, AAS 15-544, Veil, Colorado, USA, 9-13 August 2015. 
30. Shi, J. and Ghanem, R. G.: Nonlocal, Stochastic Modeling of Meterials with Multiscale Structures. Proceedings of the 6th World Congress on Computational Mechanics, Beijing, China, September 5-10 2004.

31. Smolyak S.: Quadrature and interpolation formulas for tensor products of certain classes of functions. Soviet Mathematics - Doklady 1963; 4:240-243.

32. Valli, M., Armellin, R. Di Lizia, P. and Lavagna M. R.: Nonlinear Mapping of Uncertainties in Celestial Mechanics", Journal of Guidance, Control, and Dynamics, Vol. 36, No. 1 (2013), pp. 48-63. doi: 10.2514/1.58068.

33. Sabol, C., Sukut, T., Hill, K., Alfriend, K. T., Wright, B., Li, Y., and Schumacher, P. Linearized orbit covariance generation and propagation analysis via simple Monte Carlo simulations. In Paper AAS 10-134 presented at the AAS/AIAA Space Flight Mechanics Conference, February, 14-17, (2010).

34. Vetrisano, M., van der Weg, W. and Vasile, M.: Navigating to the moon along lowenergy transfers. Celestial Mechanics and Dynamical Astronomy, vol. 114, no. 1-2, pp. 25-53, 2012. doi:10.1007/s10569-012-9436-9

35. Wiener N.: The homogeneous chaos. American Journal of Mathematics 1938; 60:897936. DOI: $10.2307 / 2371268$

36. Wittig, A., Di Lizia, P., Armellin, R., Bernelli, F., Makino, K. And Berz, M.: An Automatic Domain Splitting Technique to Propagate Uncertainties in Highly Nonlinear Orbital Dynamics. Advances in the Astronautical Sciences, Vol. 152, Univelt Inc., USA, 2014. pp. 1923-1941

37. Xiu D. and Karniadakis G.E.: The Wiener-Askey polynomial chaos for stochastic differential equations. SIAM Journal on Scientific Computing 2002; 24(2):619-644. DOI:10.1137/S1064827501387826

38. Xiu D.: Hesthaven J.S.: High-order collocation methods for differential equations with random inputs. SIAM Journal on Scientific Computing 2005; 27(3):1118-1139. DOI:10.1137/040615201 